Appl. Math. J. Chinese Univ.

$2019, * *(*): * * *_{-} * * *$

\title{
Statistical Analysis and Evaluation of Macroeconomic Policies: A Selective Review
}

\author{
LIU Ze-qin ${ }^{1}, \quad$ CAI Zong-wu²,$\quad$ FANG Ying ${ }^{1, *}, \quad$ LIN Ming ${ }^{1,3}$
}

\begin{abstract}
In this paper, we highlight some recent developments of a new route to evaluate macroeconomic policy effects, which are investigated under the framework with potential outcomes. First, this paper begins with a brief introduction of the basic model setup in modern econometric analysis of program evaluation. Secondly, primary attention goes to the focus on causal effect estimation of macroeconomic policy with single time series data together with some extensions to multiple time series data. Furthermore, we examine the connection of this new approach to traditional macroeconomic models for policy analysis and evaluation. Finally, we conclude by addressing some possible future research directions in statistics and econometrics.
\end{abstract}

Keywords. Impulse response function; Macroeconomic casual inferences; Macroeconomic policy evaluation; Multiple time series data; Potential outcomes; Treatment effect.

\section{$\S 1 \quad$ Introduction}

It is a common practice for both policymakers and scholars to perform ex post assessment of the causal effects of social-economic programs or policies or public interventions. The statistical/econometric analysis and evaluation of such causal effects has attracted considerable attention during the last two decades and has by now undergone substantial development and progress. Some scholars, for example, Imbens and Wooldridge (2009) and Cerulli (2015), provided comprehensive surveys of the existing literature. However, most of these surveys mainly focus on microeconomic policy or social program evaluations, which might not be appropriate for macroeconomic policy or intervention evaluation. There are at least three reasons on why one should pay a special attention to macroeconomic policy analysis and evaluation. First, in some macroeconomic policy evaluations, the main interest is to estimate the causal effect of

Received: **_**_**.

MR Subject Classification: 62F02, 62G02.

Keywords: Impulse response function; Macroeconomic casual inferences; Macroeconomic policy evaluation; Multiple time series data; Potential outcomes; Treatment effect.

Digital Object Identifier(DOI): ***-******.

Supported by the National Natural Science Foundation of China (71631004 (Key Project)), and the National Science Fund for Distinguished Young Scholars (71625001).

${ }^{*}$ Correspondence author. 
a policy intervention on a single country or region, rather than the average treatment effect in microeconomic policy or social program evaluations. For example, to estimate the effect of political and economic integration of Hong Kong with mainland China on Hong Kong's economic growth; see, for example, Hsiao, Ching and Wan (2012, henceforth HCW), there is only one unit, like Hong Kong, in the treated group. Therefore, in this example, only an individual treatment effect is concerned.

Second, for macroeconomic policy evaluation, it may be more difficult to find a well-matched control group for the treatment unit. For example, it is not easy to match China with other countries to assess the impact of a Chinese policy on its economy, because China has a unique economic and/or political system. Third, in a microeconomic policy evaluation setting, individual observations in different periods are usually serially autocorrelated and treatment in one period often has impacts on the following periods. Based on the above reasons, the macroeconomic policy evaluation framework should be deserved a particular consideration and developed separately. Although there are many other methods, such as dynamic stochastic general equilibrium modeling (abbreviated as DSGE) and vector autoregressive (VAR) models, which are popular to be adopted to analyze macroeconomic policy effects, most of these methods rely heavily on some structural specifications of the entire economic system. In such a way, the validity of their results relies on how precise the assumed economic models are.

The main aim of this paper is to survey recent developments of an alternative route to make causal inferences for macroeconomic policy effects. The key problem in this literature is to estimate statistically the treatment effect of a macroeconomic policy program in a nonexperimental setup. Similar to microeconomic policy evaluation, the fundamental challenge is the missing observation problem. That is, at most one outcome in different treatment levels can be observed because the unit can be exposed to only one level of the treatment. This problem is usually addressed by using the so-called counterfactual approach in the statistics literature; see, for example, Rubin $(1974,1977)$ for details, which is also called the Rubin causal model.

The rest of this paper is organized as follows. Section 2 briefly introduces the basic framework of the Rubin causal model. Section 3 reviews the estimation methods for estimating macroeconomic causal effects using single time series data. Section 4 focuses on the methods when multiple time series data are available. Section 5 concludes with discussions on some open and interesting research questions.

\section{$\S 2 \quad$ Basic Model Setup}

From the early 1970s, Rubin (1973a, 1973b, 1974, 1978, 1979), in a series of his papers, mapped the now dominant approach to the evaluation problem, labeled as Rubin causal model (RCM) by Holland (1986). There are two essential ingredients in RCM: potential outcomes and assignment mechanism. The potential outcomes framework is the hallmark of modern econometric analysis of treatment effect. This section gives a concise introduction to the basic

model setup under the RCM framework, which can be used to analyze a macroeconomic policy's 
simultaneous treatment effects under certain assumptions.

\section{$2.1 \quad$ Potential Outcomes}

Suppose that we wish to estimate the effect of a macroeconomic policy on economic growth using observations on $T$ periods, indexed by $t=1, \ldots, T$. The policy is carried out in some of these periods, not in others. Let $Y_{t}$ denote economic growth and $D_{t}$ indicate whether a policy is implemented at period $t$, with $D_{t}=1$ if it is implemented and $D_{t}=0$ if it is not. $D_{t}$ can be a binary variable, a multiple variable (see, e.g., Angrist and Imbens, 1995), or a continuous variable (Imbens, 2000, and Hirano and Imbens, 2004). If not otherwise specified, we assume $D_{t}$ is binary in this paper.

Definition 2.1 (Potential Outcomes). Potential outcomes are pairs of outcomes for the same period given different levels of treatment, denoted by $Y_{t}(0)$ and $Y_{t}(1) . Y_{t}(0)$ denotes the outcome that would be realized at period $t$ if the policy is not implemented. Similarly, $Y_{t}(1)$ denotes the outcome that would be realized at period $t$ if the policy is implemented.

Rubin (1973a, 1973b) defined novelly the causal effect as the difference between this pair of outcomes, i.e., $Y_{t}(1)-Y_{t}(0)$, the treatment effect of the policy at period $t$. This framework counters a fundamental missed observation problem, since at period $t$, the policy can either be implemented or not be implemented, but not both. Thus, only one of these two potential outcomes can be realized. However, prior to the assignment being determined, both are potentially observable and hence labeled as potential outcomes. If the policy is carried out at period $t, Y_{t}(1)$ is realized, and $Y_{t}(0)$ is a counterfactual outcome. On the other hand, if the policy is not carried out at period $t, Y_{t}(0)$ is realized, and $Y_{t}(1)$ is a counterfactual outcome. In casual inference, it is usual to make few assumptions other than the stable unit treatment value assumption (SUTVA), which is that one unit's outcomes are unaffected by another unit's treatment assignment.

The potential outcomes $Y_{t}(0)$ and $Y_{t}(1)$ have the following relationship with the realized outcome $Y_{t}$ :

$$
Y_{t}=\left\{\begin{array}{ll}
Y_{t}(0), & D_{t}=0 ; \\
Y_{t}(1), & D_{t}=1 .
\end{array} \quad \text { Equivalently, } \quad Y_{t}=D_{t} Y_{t}(1)+\left(1-D_{t}\right) Y_{t}(0),\right.
$$

which is the so-called potential outcome model (POM), and it is the fundamental relation linking unobservable and observable outcomes. The potential outcomes framework has five main advantages over the traditonal models defined by realized outcomes; see Imbens and Wooldridge (2009) for more details.

\section{$2.2 \quad$ Parameters of Interest}

Average treatment effect (ATE) and average treatment effect on the treated (ATET) are two prominent parameters of interest in the literature. If $Y_{t}$ and $D_{t}$ is strictly stationary, then

$$
\mathrm{ATE}=E\left[Y_{t}(1)-Y_{t}(0)\right], \quad \text { and } \quad \mathrm{ATET}=E\left[Y_{t}(1)-Y_{t}(0) \mid D_{t}=1\right] .
$$


Average treatment effect is defined over the entire period, and average treatment effect on the treated is averaged over the subset of treated periods. Another popular parameter is conditional average treatment effects (CATE):

$$
\operatorname{ATE}(\mathbf{x})=E\left[Y_{t}(1)-Y_{t}(0) \mid \mathbf{X}_{t}=\mathbf{x}\right], \quad \text { and } \quad \operatorname{ATE}=E_{\mathbf{x}}[\operatorname{ATE}(\mathbf{x})]
$$

where $\mathbf{X}_{t}$ is a vector of conditional variables (strictly stationary, and not affected by the intervention). Recently, a quantile treatment effect (QTE) has been popularly studied and extensively applied in the economics literature and real applications; see Abadie, Angrist and Imbens (2002), Chernozhukov and Hansen (2005), and Koenker and Bassett (1978). For a given $0<q<1$, QTE is defined as

$$
\mathrm{QTE}_{q}=F_{Y(1)}^{-1}(q)-F_{Y(0)}^{-1}(q),
$$

where $F_{Y(d)}(\cdot)$ is the distribution of $Y_{t}(d)$ for $d=1$ and 0 . The conditional quantile treatment effect (CQTE) and conditional quantile treatment effect on the treated (CQTET) can be defined in the same way.

\subsection{Assignment Mechanism}

The assignment mechanism is the mechanism by which policies are carried out or not at period time $t$. It is expressed as the conditional probability of receiving the treatment given observed covariates and potential outcomes, i.e., $P\left(D_{t}=1 \mid \mathbf{X}_{\mathbf{t}}, Y_{t}(0), Y_{t}(1)\right)$, which is a function of potential outcomes and observed covariates. According to the relationship between potential outcomes and the assignment mechanism, three classes of assignment mechanism are distinguished commonly as: randomized experiments, selection on observables, and selection on unobservables, which are described next in detail.

The first class of assignment mechanisms is randomized experiments. In randomized experiments, $D_{t} \Perp(Y(0), Y(1))$, i.e., the probability of assignment to treatment, a known function of covariates, is independent with potential outcomes. A typical example is a completely randomized experiment where we randomly choose $T_{1}<T$ periods for treated and $T_{0}=T-T_{1}$ for untreated, and each period has equal probability of being in the control group or the treatment group. Under the assumptions of iid (independently and identically distributed) and SUTVA, the well-known difference-in-means (DIM) estimator in classical statistics defined in (3) can be applied to recover the average treatment effect:

$$
\widehat{\mathrm{DIM}}=\frac{1}{T_{1}} \sum_{t=1}^{T_{1}} D_{t} Y_{t}-\frac{1}{T_{0}} \sum_{t=T_{1}+1}^{T}\left(1-D_{t}\right) Y_{t} .
$$

In reality, the decision-making process for a macroeconomic policy is complex. To carry out a

policy, the authority would gather detailed and well-documented data and information, outline the problems, list goals and objectives, reveal potential gains and losses, etc. Thus, it is impossible for the authority to implement a macroeconomic policy randomly.

The second class of assignment mechanisms is selection on observables, which is also referred to as unconfounded assignment, which was first presented by Rosenbaum and Rubin (1983). This mechanism assumes that factors determining whether a policy should be put into effect 
are observable. In this setting, $D_{t} \Perp\left(Y_{t}(0), Y_{t}(1)\right) \mid \mathbf{X}_{t}$, i.e., given $\mathbf{X}_{t}$, the assignment probability does not depend on the potential outcomes. Different from randomized experiments, the assignment probability is no longer assumed to be a known function of covariates.

Thus far, a majority of statistical/econometric methods for evaluation have been developed in such a setup under the assumptions of iid and SUTVA. The most popular techniques in this vein include, but not limited to, regression-adjustment (RA) in Heckman et al. (1997, 1998), matching in Abadie and Imbens (2006, 2008, 2011), matching on propensity-score in Abadie and Imbens (2016), Dehejia and Wahba (2002), and Caliendo and Kopeinig (2010), re-weighting on propensity-score inverse probability (IPW) in Rosenbaum and Rubin (1983), Dehejia and Wahba (1999), Hahn (1998), Hirano, Imbens and Ridder (2003), Brunell and DiNardo (2004), Wooldridge (2010), Imbens (2004), and Lunceford and Davidian (2004), and double-roust estimator (DR) in Robins and Rotnitzky (1995), Robins, Rotnitzky and Zhao (1995), van der Lann and Robins (2003), and Wooldridge (2007), and the references therein.

Finally, the third one is selection on unobservables, containing cases apart from the random experiment and selection on observables. In this setting, whether a policy is implemented depends on not only observable factors but also unobservable factors. Thus, the assignment probability has some dependence on potential outcomes. Therefore, given $\mathbf{X}_{t}$, we cannot identify the treatment effects, and we require further assumptions for the analysis. There is no general solution for this situation.

\section{$\S 3$ Evaluation Methods With Single Time Series Data}

The model setup in section 2 is borrowed from microeconomic policy evaluation but is still suitable for assessing a macroeconomic policy's simultaneous effects on outcome variables with some additional assumptions. However, subsequent movements of the outcome variables responding to the policy are needed for a careful consideration in a macroeconomic policy evaluation framework. Angrist and Kuersteiner (2011), Angrist, Jordà and Kuersteiner (2018), Bojinov and Shephard (2017), Jordà and Taylor (2016), and Kuersteiner, Phillips and VillamizarVillegas (2018) extended the modern statistical/econometric analysis of treatment effects to a time series context for macroeconomic data.

\subsection{A Road Map}

In cross-sectional analysis, we have data usually consisting of multiple units in one period, some being treated but others not. Since we cannot observe the pairs of potential outcomes simultaneously, the individual treatment effect cannot be identified. By comparing the outcomes of treatment group with control group, we obtain the consistent estimation of average treatment effect. However, in time series analysis, we only have data of a single unit in multiple periods. Instead of the average treatment effect, the concepts of potential outcome paths introduced by Robins (1986) and the dynamic potential outcomes become the heart of this literature. 


\subsubsection{Potential Outcome Paths}

Let $D_{1: t}=\left(D_{1}, \cdots, D_{t}\right)$ denote the random "treatment path" and $d_{1: t}$ be a realization of $D_{1: t}$. By assuming that $D_{t}$ is binary, at time $t$, we have $2^{t}$ treatment paths. Up to time $t$, we have a set of $2\left(2^{t}-1\right)$ treatment paths (see Figure 1 below), while Bojinov and Shephard (2017) defined potential outcomes on treatment path $D_{1: t}$, not $D_{t}$ as Section 2.

Definition 3.1 (Potential Outcome Paths). The set of $2^{t}$ potential outcomes at time $t$ is:

$$
Y_{t}(\cdot)=\left\{Y_{t}\left(d_{1: t}\right): d_{1: t} \in\{0,1\}^{t}\right\},
$$

the collection of $2\left(2^{t}-1\right)$ potential outcome paths up to time $t$ is:

$$
Y_{1: t}(\cdot)=\left\{Y_{1}(\cdot), Y_{2}(\cdot), \cdots, Y_{t}(\cdot)\right\},
$$

and the potential path for the treatment path $d_{1: t}$ is

$$
Y_{1: t}\left(d_{1: t}\right)=\left\{Y_{1}\left(d_{1: 1}\right), Y_{2}\left(d_{1: 2}\right), \cdots, Y_{t}\left(d_{1: t}\right)\right\} .
$$

Therefore, the potential outcome paths have the following relationship with observed outcome path:

$$
Y_{1: t}=\sum_{d \in\{0,1\}^{t}} 1_{d_{1: t}=d} Y_{1: t}(d), \quad t=1, \cdots, T .
$$

To see the detailed paths of potential outcome, let us consider the case that $T=3$ with the potential outcome paths indicated in Figure 1 . When $t=1$, there are $2^{1}=2$ poten-

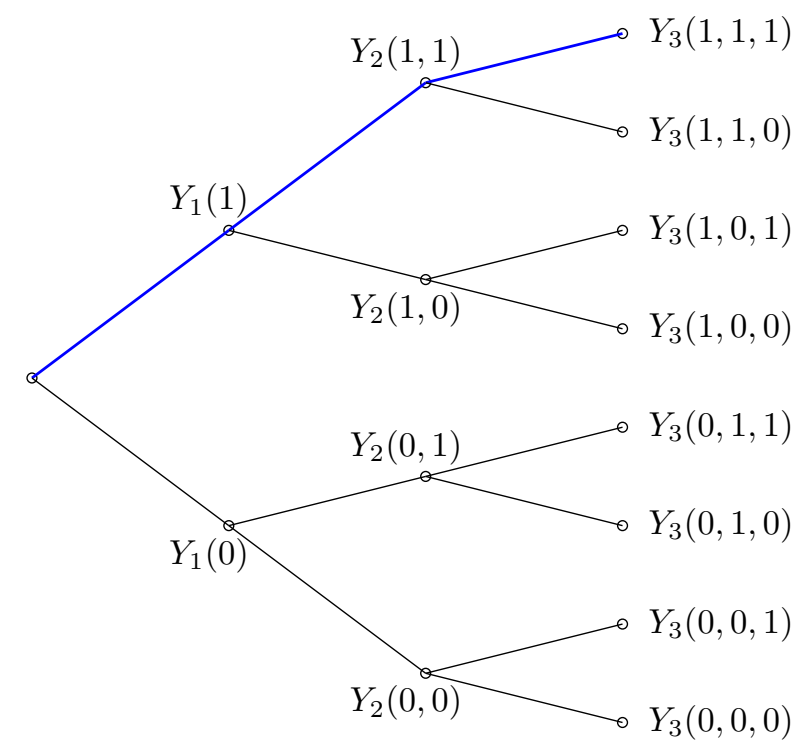

Figure 1: Potential outcome paths when $\mathrm{T}=3$

tial outcomes $Y_{1}(0)$ and $Y_{1}(1)$. When $t=2$, there are $2^{2}=4$ potential outcomes $Y_{2}(1,1)$, $Y_{2}(1,0), Y_{2}(0,1)$, and $Y_{2}(0,0)$. Up to $t=2$, there are $2 \cdot\left(2^{2}-1\right)=6$ potential paths set $\left\{Y_{1}(0), Y_{1}(1), Y_{2}(1,1), Y_{2}(1,0), Y_{2}(0,1), Y_{2}(0,0)\right\}$. Assuming that the realized treatment path 
is $d_{1: 3}=(1,1,1)$, then, the potential path for $d_{1: 3}$ is $Y_{1: 3}\left(d_{1: 3}\right)=\left\{Y_{1}(1), Y_{2}(1,1), Y_{3}(1,1,1)\right\}$. According to (4), the observed outcome path is just the potential outcome path of observed treatment path $d_{1: t}$, when $\mathrm{t}=3, Y_{1: t}=Y_{1: t}\left(d_{1: t}\right)=\left\{Y_{1}(1), Y_{2}(1,1), Y_{3}(1,1,1)\right\}$.

Given the potential outcome paths, any comparison of potential outcome paths at fixed period $t$, has a causal interpretation. Therefore, we can define a large number of treatment effects; see, for example, Bojinov and Shephard (2017).

Definition 3.2 (General Treatment Effect). For paths $d_{1: t}$ and $d_{1: t}^{\prime}$, the $t$-th treatment effect is:

$$
\tau_{t}\left(d_{1: t}, d_{1: t}^{\prime}\right)=Y_{t}\left(d_{1: t}\right)-Y_{t}\left(d_{1: t}^{\prime}\right)
$$

and the average treatment effect of the paths $d_{1: t}$ and $d_{1: t}^{\prime}$ is:

$$
\bar{\tau}\left(d_{1: t}, d_{1: t}^{\prime}\right)=\frac{1}{T} \sum_{t=1}^{T} \tau_{t}\left(d_{1: t}, d_{1: t}^{\prime}\right) .
$$

\subsubsection{Dynamic Potential Outcomes}

In the DSGE and VAR frameworks, one of the main focuses is the impulse response function (IRF), which is the contemporaneous and subsequent movements of outcome variables responding to an exogenous policy shock at time $t$. Angrist and Kuersteiner (2011) and Angrist et al. (2018) proposed the new concept of dynamic treatment effects as the corresponding part of the impulse response function.

Definition 3.3 (Dynamic Potential Outcomes). Given $t, l$, and $\psi$, potential outcomes $\left\{Y_{t, l}^{\psi}(d) ; d \in\right.$ $\mathcal{D}$ \} are defined as the set of values the observed outcome variable $Y_{t+l}$ would take on if $D_{t}=d$, with $d \in \mathcal{D}=\left\{d_{0}, \cdots, d_{j}, \cdots, d_{J}\right\} . \psi$ denotes the policy regime, which takes values in a parameter space $\Psi$.

Let $Y_{t: L}=\left(Y_{t+1}, \cdots, Y_{t+L}\right)^{\prime}$ is the path of the observed outcome from $\mathrm{t}+1$ to $\mathrm{t}+\mathrm{L}, Y_{t: L}^{\psi}(d)=$ $\left(Y_{t, 1}^{\psi}(d), \cdots, Y_{t, L}^{\psi}(d)\right)^{\prime}$ is the path of potential outcome from $\mathrm{t}+1$ to $\mathrm{t}+\mathrm{L}$. The relationship of $Y_{t: L}$ and $Y_{t: L}^{\psi}(d)$ is:

$$
Y_{t: L}=\sum_{d \in \mathcal{D}} Y_{t: L}^{\psi}(d) 1\left\{D_{t}=d\right\}
$$

Based on the definition above, the causal effect of policy choice $d_{j}$ is $Y_{t, l}^{\psi}\left(d_{j}\right)-Y_{t, l}^{\psi}\left(d_{0}\right)$, where $d_{0}$ is a benchmark policy. Since individual causal effects can never be observed, Angrist and Kuersteiner (2011) and Angrist et al. (2018) thus defined the average treatment effects, which are labelled as the dynamic treatment effects.

Definition 3.4 (Dynamic Treatment Effects). Given l, the expected response of $Y_{t+l}$ to policy $d_{j}$ is:

$$
\theta_{l, j}=E\left[Y_{t, l}^{\psi}\left(d_{j}\right)-Y_{t, l}^{\psi}\left(d_{0}\right)\right],
$$

and the collection of treatment effects from $t+1$ to $t+L$ is:

where $\theta_{j}=\left(\theta_{1, j}, \cdots, \theta_{L, j}\right)$.

$$
\theta_{j}=E\left[Y_{t: L}^{\psi}\left(d_{j}\right)-Y_{t: L}^{\psi}\left(d_{0}\right)\right]
$$


Similar to the traditional impulse response analysis in macroeconomics, the definition for $\theta$ focuses on a single policy shock on subsequent outcomes. Indeed, it compares two different paths, which control the path of $D_{t}$ before $t$, and assume $d_{t+1: t+L \mid t}$ is constant:

$$
\theta_{l, j}\left(d_{j}, d_{0}\right)=E\left[Y_{t, l}^{\psi}\left(d_{1: t-1}, d_{t}=d_{j}, d_{t+1}, \cdots, d_{t+l}\right)-Y_{t, l}^{\psi}\left(d_{1: t-1}, d_{t}=d_{0}, d_{t+1}, \cdots, d_{t+l}\right)\right],
$$

and

$$
\theta_{j}\left(d_{j}, d_{0}\right)=E\left[Y_{t: L}^{\psi}\left(d_{1: t-1}, d_{t}=d_{j}, d_{t+1}, \cdots, d_{t+L}\right)-Y_{t: L}^{\psi}\left(d_{1: t-1}, d_{t}=d_{j}, d_{t+1}, \cdots, d_{t+L}\right)\right] .
$$

Note that both the timing of policy adoption and the horizon matter for $Y_{t, l}^{\psi}\left(d_{j}\right)$. For example, $Y_{t, l}^{\psi}\left(d_{j}\right)$ may be different from $Y_{t+1, l-1}^{\psi}\left(d_{j}\right)$, even though both describe outcomes in period $t+l$, because $Y_{t, l}^{\psi}\left(d_{j}\right)$ measures the effect of $D_{t}=d_{j}$ on the outcome at time $t+l, Y_{t+1, j-1}^{\psi}\left(d_{j}\right)$ measures the effect of $D_{t+1}=d_{j}$ on the outcome at time $t+l$, and for $Y_{t, l}^{\psi}\left(d_{j}\right)$, we do not limit $D_{t+1}=d_{j}$.

\subsection{Connection to Classic Macroeconomic Models}

To understand how the dynamic treatment effect defined above corresponds to a nonlinear impulse response function, we illustrate their connection using the following model:

$$
D_{t}=D\left(z_{t}, \psi, \epsilon_{t}\right), \quad \text { and } \quad Y_{t+l}=F_{t, l}\left(z_{t}, D_{t}, \eta_{t, l}\right),
$$

where $z_{t}$ is a vector including covariate $\mathbf{X}_{t}$, as well as some lags of $Y_{t}$ and $D_{t}, \epsilon_{t}$ is unobservable idiosyncratic information, which is assumed to be independent of potential outcomes, and $\eta_{t, l}$ is white noise. Considering a perturbation $\epsilon_{t}$ of $D_{t}$ at time $t$, the nonlinear impulse response of $Y_{t+l}$ is defined as:

$$
\delta_{l}\left(z_{t}, D_{t}, \epsilon_{t}\right)=F_{t, l}\left(z_{t}, D\left(z_{t}+\epsilon_{t}\right), \eta_{t+l}\right)-F_{t, l}\left(z_{t}, D\left(z_{t}\right), \eta_{t+l}\right) .
$$

If $D\left(z_{t}+\epsilon_{t}\right)=d_{j}$, and $D\left(z_{t}\right)=d_{0}$, it is clear that (7) is just the dynamic treatment effect defined above:

$$
\theta_{l, j}=E\left[Y_{t, l}^{\psi}\left(d_{j}\right)-Y_{t, l}^{\psi}\left(d_{0}\right)\right] .
$$

Therefore, Angrist and Kuersteiner (2011) made this strong link to linear impulse response function in their paper. Suppose in a VAR model,

$$
\Gamma_{0}\left[\begin{array}{c}
Y_{t} \\
D_{t} \\
\mathbf{X}_{t}
\end{array}\right]=-\Gamma(L)\left[\begin{array}{c}
Y_{t} \\
D_{t} \\
\mathbf{X}_{t}
\end{array}\right]+\left[\begin{array}{c}
\eta_{t} \\
\epsilon_{t} \\
\zeta_{t}
\end{array}\right],
$$

where $\mathrm{L}$ is the lag operator, $\epsilon_{t}$ represents the policy shocks in which we are interested, $\eta_{t}$ and $\zeta_{t}$ represents other innovations. Assume $C(L)=\left(\Gamma_{0}+\Gamma(L)\right)^{-1}$ exists, then,

$$
\left[\begin{array}{c}
Y_{t} \\
D_{t} \\
\mathbf{X}_{t}
\end{array}\right]=C(L)\left[\begin{array}{l}
\eta_{t} \\
\epsilon_{t} \\
\zeta_{t}
\end{array}\right]
$$

We further suppose that the outcome is determined by $Y_{t}=\phi_{1} \epsilon_{t}+\phi_{2} D_{t}+\phi_{3} \eta_{t}$, and the policy rule is given by $D_{t}=\psi Y_{t-1}+\epsilon_{t}$, where $Y_{t}$ has a moving average representation:

$$
Y_{t}=\sum_{l=0}^{\infty} \rho_{l} \epsilon_{t-j}+\sum_{l=0}^{\infty} \gamma_{l} \eta_{t-l} .
$$


Here, $\rho_{l}$ is the impulse response function for output in response to the interested policy shocks. In this setup, potential outcome for $D_{t}=d_{j}$ is defined as:

$$
Y_{t, l}^{\psi}\left(d_{j}\right)=\rho_{j}\left(d_{j}-\psi Y_{t-1}\right)+\sum_{j=0, j \neq l}^{\infty} \rho_{j} \epsilon_{t+l-j}+\sum_{j=0}^{\infty} \gamma_{j} \eta_{t-j} .
$$

The associated causal effect of this policy change is $Y_{t, l}^{\psi}\left(d_{j}\right)-Y_{t, l}^{\psi}\left(d_{0}\right)=\rho_{j}\left(d_{j}-d_{0}\right)$, which means how the outcome $Y_{t+j}$ would change if the policy changing from $d_{0}$ to $d_{j}$, by assuming that everything else is unchanged. Obviously, this is precisely the idea of the impulse response function. Moreover, compared to DSGE models, the identification and estimation of dynamic treatment effects requires no need to specify the structural process of $Y_{t}$ and only focuses on the policymaking process, alleviating the crucial model misspecification problem faced in the other main macroeconomic models and providing a more flexible tool for the analysis and evaluation of macroeconomic causal relationships.

\subsection{Inferences With Selection on Observables}

Selection on observables is a natural starting point for identification and estimation of the causal inferences. Angrist and Kuersteiner (2011) and Angrist et al. (2018) defined the assumption of selection on observables in time series framework as follows:

Assumption 3.1 (Selection on Observables). Given covariate $z_{t}, Y_{t, l}^{\psi}\left(d_{j}\right) \Perp D_{t} \mid z_{t}$ for all $l \geq 0, d_{j}$, with $\psi$ fixed, and $\psi \in \Psi$.

This assumption is also known as conditional independence assumption (CI). It also can be expressed as the following form:

$$
P\left(D_{t}=d_{j} \mid D_{1: t-1}=d_{1: t-1}, Y_{1: T}^{\psi}(\cdot), z_{t}\right)=P\left(D_{t}=d_{j} \mid D_{1: t-1}=d_{1: t-1}, Y_{1: t-1}^{\psi}\left(d_{1: t-1}\right), z_{t}\right) .
$$

In view of (8), it is clear see that the assumption means that our treatments only depend on past observables of potential outcomes and are not influenced by the corresponding period value or by future values of potential outcomes. This assumption is simply the "sequential randomization" assumption as in Robins (1994), Robins, Greenland and Hu (1999), Abbring and van den Berg (2003), and Lok (2008). Under this assumption, we have the following relationship:

$$
\theta_{l, j}=E\left[E\left[Y_{t, l}^{\psi}\left(d_{j}\right)-Y_{t, l}^{\psi}\left(d_{0}\right) \mid z_{t}\right]\right]=E\left[E\left[Y_{t, l} \mid D_{t}=d_{j}, z_{t}\right]-E\left[Y_{t, l} \mid D_{t}=d_{0}, z_{t}\right]\right] .
$$

Then, $\theta_{l, j}$ can be identified, since (9) is cast in terms of observable conditional means. Therefore, to estimate $\theta_{l, j}$, we can draw lessons from the iid case. Indeed, Angrist et al. (2018) applied the IPW estimator to time series data to estimate the causal effect of monetary policy on macroeconomic aggregates, while Jordà and Taylor (2016) adopted the doubly robust estimator to time series data to evaluate the fiscal policy. They are elaborated in detail in the next subsections. 


\subsubsection{Estimation: IPW Method}

Define $p^{j}\left(z_{t}, \psi\right)=P\left(D_{t}=d_{j} \mid z_{t}\right)$ as the policy propensity score, with $p^{j}\left(z_{t}, \psi\right)$ being a flexible parametric model with parameter $\psi$ determined by the policy regime, where $z_{t}$ is a covariate. Further, assume that $0<\underline{p} \leq p^{j}\left(z_{t}, \psi\right) \leq \bar{p}<1$, which is a common assumption in the literature. Then, the selection on observables assumption implies that

which leads to

$$
E\left[Y_{t, l} 1\left\{D_{t}=d_{j}\right\} \mid z_{t}\right]=E\left[Y_{t, l}^{\psi}\left(d_{j}\right) \mid z_{t}\right] p^{j}\left(z_{t}, \psi\right)
$$

$$
\theta_{l, j}^{I P W}=E\left[Y_{t, l}\left(\frac{1\left\{D_{t}=d_{j}\right\}}{p^{j}\left(z_{t}, \psi\right)}-\frac{1\left\{D_{t}=d_{0}\right\}}{p^{0}\left(z_{t}, \psi\right)}\right)\right] .
$$

This re-weighting scheme was first proposed by Horvitz and Thompson (1952) and was adapted by Hanh (1998) and Hirano et al. (2003) for treatment effect estimation in cross-sectional cases.

Denote

$$
w_{t, j}(\psi)=\frac{1\left\{D_{t}=d_{j}\right\}}{p^{j}\left(z_{t}, \psi\right)}-\frac{1\left\{D_{t}=d_{0}\right\}}{p^{0}\left(z_{t}, \psi\right)} .
$$

Then, (10) can be written as $\theta_{l, j}^{I P W}=E\left[w_{t, j}(\psi) Y_{t, l}\right]$, a weighted expectation of $Y_{t, l}$, and clearly, $\theta_{l, j}^{I P W}$ can be estimated by a two-step procedure. First, estimate the parameter $\psi$ in $p^{j}\left(z_{t}, \psi\right)$. Second, take $\hat{\psi}$ to $w_{t, j}(\hat{\psi})$ and then obtain the estimation of $\theta_{l, j}^{I P W}$. In a correctly specified model, the weight $w_{t, j}(\psi)$ has a mean zero and is uncorrelated with $z_{t}$. To ensure this condition is true, Angrist et al. (2018) suggested weight $Y_{t, l}$ by $\ddot{w}_{t, j}$, where $\ddot{w}_{t, j}=w_{t, j}(\hat{\psi})-\hat{w}_{t, j}$, which is the residual from a regression of $w_{t, j}(\hat{\psi})$ on $z_{t}$ and a constant. Therefore, the estimators of $\theta_{l, j}^{I P W}$ and $\theta_{j}^{I P W}$ are given by

$$
\hat{\theta}_{l, j}^{I P W}=\frac{1}{T} \sum_{t=1}^{T} \ddot{w}_{t, j} Y_{t+l}, \quad \text { and } \quad \hat{\theta}_{j}^{I P W}=\frac{1}{T} \sum_{t=1}^{T} \ddot{w}_{t, j} Y_{t: L},
$$

respectively. In practice, when $p^{j}\left(z_{t}, \hat{\psi}\right)$ is too small, the weight would be extremely large. Then, the estimation of $\theta_{l, j}^{I P W}$ is dominated by these outliers. To overcome this difficulty, the trimmed method should be used by dropping observations with $p^{j}\left(z_{t}, \hat{\psi}\right)<0.025$ when $1\left\{D_{t}=d_{j}\right\}=1$. Note that when the dimension of $z_{t}$ is not very high, the nonparametric estimate of $p^{j}\left(z_{t}\right)$ can be applied to avoid a possible misspecification of parametric form $p^{j}\left(z_{t}, \psi\right)$.

\subsubsection{Estimation: Doubly Robust Approch}

It is well documented in the literature that if the parametric form of $p^{j}\left(z_{t}, \psi\right)$ is correctly specified, the IPW estimators $\hat{\theta}_{l, j}^{I P W}$ and $\hat{\theta}_{l}^{I P W}$ are consistent. To circumvent the possible misspecification of $p^{j}\left(z_{t}, \psi\right)$, one can let $p^{j}\left(z_{t}\right)=P\left(D_{t}=d_{j} \mid z_{t}\right)$ be unspecified as a nonparametric form so that the IPW estimators are consistent. However, the dimension of $z_{t}$ is high in practice, the nonparametric estimate of $p^{j}(\cdot)$ may suffer from the "curse of dimensionality". To overcome these difficulties, Robins and Rotnitzky (1995) proposed the so-called doubly robust estimator (DR).

Jordà and Taylor (2016) adopted the doubly robust estimator to assess the impact of the U.K. government's fiscal austerity policies in 2010 on subsequent economic growth. The doubly robust estimator has an attractive property that consistency of the estimated average treatment 
effect only requires either the propensity score model or the regression model of the potential outcome to be correctly specified.

The doubly robust estimator used by Jordà and Tapylor (2016) is indeed an AIPW (augmented IPW), which has the smallest asymptotic variance within the doubly robust class as in Robins and Rotnitzky (1995), Robins et al. (1995), and Lunceford and Davidian (2004), and it is defined by

$$
\begin{aligned}
\theta_{l, j}^{A I P W}=\frac{1}{T} \sum_{t=1}^{T}\left[\frac{1\left\{D_{t}=d_{j}\right\}}{p^{j}\left(z_{t}, \hat{\psi}\right)} Y_{t+l}+\left(1-\frac{1\left\{D_{t}=d_{j}\right\}}{p^{j}\left(z_{t}, \hat{\psi}\right)}\right) \mu_{j}\left(z_{t}, \hat{\beta}_{j}\right)\right] \\
-\frac{1}{T} \sum_{t=1}^{T}\left[\frac{1\left\{D_{t}=d_{0}\right\}}{p^{0}\left(z_{t}, \hat{\psi}\right)} Y_{t+l}+\left(1-\frac{1\left\{D_{t}=d_{0}\right\}}{p^{0}\left(z_{t}, \hat{\psi}\right)}\right) \mu_{0}\left(z_{t}, \hat{\beta}_{0}\right)\right]
\end{aligned}
$$

where $\mu_{j}\left(z_{t}, \hat{\beta}_{j}\right)=E\left(Y_{t+l} \mid D_{t}=d_{j}, z_{t}\right)$ and $\mu_{0}\left(z_{t}, \hat{\beta}_{j}\right)=E\left(Y_{t+l} \mid D_{t}=d_{0}, z_{t}\right)$, which are two regression estimators of potential outcomes $Y_{t, l}^{\psi}\left(d_{j}\right)$ and $Y_{t, l}^{\psi}\left(d_{0}\right)$, respectively.

The AIPW estimator is the basic IPW estimator plus an adjustment of the weighted average of the two regression estimators. The adjustment term stabilizes the estimator when the propensity scores become close to zero or one (Glynn and Quinn, 2010). In the cross-sectional context, when the propensity score and the regression function are modeled correctly, the AIPW can achieve the semiparametric efficiency bound. If the propensity score $p^{j}(\cdot)$ is modeled correctly, the AIPW estimator has the asymptotic variance smaller than or equal to that for the simple IPW estimator. If the regression model $\mu_{j}(\cdot)$ is modeled correctly, the AIPW estimator should have the asymptotic variance greater than or equal to that for the simple regression estimator, but it gives protection in the event that $\mu_{j}(\cdot)$ is misspecified.

\subsubsection{Estimation: IPW Combined With Machine Learning}

Cai, Fang and Liu (2019) combined machine learning with IPW to investigate the separate and concurrent effects of monetary policy and macro-prudential policy on financial stability and economic growth in China. The motivation of Cai et al. (2019) is based on the finding in Kang and Schafer (2007) which says that IPW estimator is sensitive to misspecification of the propensity score model, especially when some estimated propensity scores are small. Kang and Schafer (2007) also showed that two wrong models in doubly robust estimators are not better than one. To alleviate these problems, Cai et al. (2019) suggested using a vast set of economic variables to span the unobservable information sets from policy makers as far as possible to estimate policy propensity score. First, they collected 158 macro economic monthly series, and then, retained 58 variables after correlation analysis.

Cai et al. (2019) adopted methods of machine learning to deal with estimation problems with large set of covariates. Pedregosa et al. (2011) did simulations and found that due to directly optimizing log-loss function, logistic regression model return well calibrated probability predictions, while other machine learning methods return biased probabilities, with different biases per method, while Niculescu-Mizil and Caruana (2007) pointed that methods like bagging and random forests average predictions from a base set of models, thus variance in the underlying 
base models would lead to biased predictions near 0 and 1 . Therefore, different from the aforementioned literature, Cai et al. (2019) adopted penalized logistic regression model to estimate policy propensity score. Define the model of $p^{j}\left(z_{t}, \psi\right)=P\left(D_{t}=d_{j} \mid z_{t}\right)$ as:

$$
p^{j}\left(z_{t}, \psi\right)=\frac{e^{\psi_{j} z_{t}}}{\sum_{k=0}^{J} e^{\psi_{k} z_{t}}}, \quad j=0,1, \cdots, J,
$$

where $z_{t}$ is lags of these 58 variables. By adding $\mathrm{L}_{1}$ type or $\mathrm{L}_{2}$ type penalty, $\hat{\psi}$ is estimated to minimize the following objective function:

$$
L_{q}(\psi, \lambda)=-\frac{1}{T}\left[\sum_{t=1}^{T} \sum_{j=0}^{J} 1\left\{D_{t}=d_{j}\right\}\left(\psi_{j} z_{t}-\log \sum_{k=0}^{J} e^{\psi_{k} z_{t}}\right)\right]+\lambda_{1}\|\psi\|_{q}^{q},
$$

where $\|\psi\|_{q}$ is the $\mathrm{L}_{q}$ normal with, say, $q=1$ or 2 . Through utilizing the information from a large number of series and eliminating the arbitrary reliance on a small number of predefined variables, Cai et al. (2019) found that the penalized logistic regression models can predict the Chinese government's policy decision precisely, especially the model with $\mathrm{L}_{2}$ penalty, which gives better predictions of policies.

With policy propensity score estimated by logistic regression model with $\mathrm{L}_{2}$ penalty, Cai et al. (2019) estimated the dynamic treatment effects of monetary policy and macro-prudential policy on financial stability and economic growth in China according to (10). The findings are that easy monetary policy alone could reduce financial systematic risk, while tight monetary policy and the combination of tight monetary policy and tight macro-prudential policy could increase financial systematic risk and slow down the economic growth rate.

\subsubsection{Testing for The Selection on Observables Assumption}

As one can see from (9) that the selection on observables assumption plays a vital role in identifying the parameters of interest $\theta_{l, j}$. However, this assumption may be violated in practice if there exist unobserved confounders which affect both the potential outcomes $Y_{t, l}^{\psi}\left(d_{j}\right)$ and the treatment variable $D_{t}$. If this assumption does not hold, both the IPW and doubly robust estimators discussed above are inconsistent estimation in general for the parameters of interest $\theta_{l, j}$. Thus, it is desirable to have formally a procedure to test whether the selection on observables assumption holds or not. Fortunately, there are some researches in the literature to propose some procedures to test whether this assumption is true or not under some frameworks. For example, Donald, Hsu and Lieli (2014) proposed a Durbin-Wu-Hausman type statistic to test the conditional mean independence, which was a weaker version of the selection on observables assumption, by using additional binary instrumental variables and Chen, Ji, Zhou and Zhu (2017) suggested another conditional mean independence test without relying on the availability of the binary instrumental variables but requiring some other conditions in the cross-sectional data setting; see Donald et al. (2014) and Chen et al. (2017) for more details. Recently, Cai, Fang, Lin and Tang (2019) proposed an alternative method to test the selection on observables assumption for both cross-sectional and time series data settings. Specifically, their method relies on the existence of an auxiliary variable which is correlated to potential 
outcomes but is independent of the treatment variable given on potential outcomes and some other observable covariates. Formally, their procedure requires the available auxiliary variable $\omega_{t}$ satisfying the following assumption.

Assumption 3.2. There exists an auxiliary variable $\omega_{t}$ such that $\omega_{t} \Perp D_{t} \mid\left(Y_{t, l}^{\psi}\left(d_{j}\right), z_{t}\right)$ for all $l \geq 0, d_{j}$, with $\psi$ fixed, and $\psi \in \Psi$.

Under this assumption, Cai et al. (2019) showed that the selection on observables assumption implied that $E\left(D_{t} \mid \omega_{t}, z_{t}\right)=E\left(D_{t} \mid z_{t}\right)$, so that testing for the selection on observables assumption could be transformed into testing the insignificance of the auxiliary variable $\omega_{t}$, which could be formulated as the following testing hypothesis

$$
H_{0}: E\left(D_{t} \mid \omega_{t}, z_{t}\right)=E\left(D_{t} \mid z_{t}\right)
$$

a.e. (almost surely) versus

$$
H_{1}: E\left(D_{t} \mid \omega_{t}, z_{t}\right) \neq E\left(D_{t} \mid z_{t}\right) \text { on a set with positive measure. }
$$

Let $\varpi_{t}=\left(z_{t}^{\prime}, \omega_{t}^{\prime}\right)^{\prime} \in \mathbb{R}^{p}$, where $p=d+r$ with $d$ being the dimension of $z_{t}$ and $r$ being the dimension of $\omega_{t}$. Define $\varepsilon_{t}=D_{t}-E\left(D_{t} \mid z_{t}\right)$. Then, the null hypothesis can be rewritten as

$$
H_{0}: E\left(\varepsilon_{t} \mid \varpi_{t}\right)=0 \text { a.e. }
$$

and the alternative hypothesis is

$$
H_{1}: E\left(\varepsilon_{t} \mid \varpi_{t}\right) \neq 0 \text { on a set with positive measure. }
$$

Furthermore, based on the following conditional moment

$$
S=E\left[\varepsilon_{t} f\left(z_{t}\right) \cdot E\left(\varepsilon_{t} f\left(z_{t}\right) \mid \varpi_{t}\right) \cdot f_{\varpi}\left(\varpi_{t}\right)\right],
$$

where $f\left(z_{t}\right)$ and $f_{\varpi}\left(\varpi_{t}\right)$ are the density functions of $z_{t}$ and $\varpi_{t}$, respectively, Cai et al. (2019) proposed the following test statistic

$$
S_{T}=\frac{1}{T(T-1) h^{p}} \sum_{t=1}^{T} \sum_{s \neq t}\left(\hat{\varepsilon}_{t} \hat{f}\left(z_{t}\right) \cdot\left(\hat{\varepsilon}_{s} \hat{f}\left(z_{s}\right)\right)\right) K_{t s}
$$

where $\hat{\varepsilon}_{t}=D_{t}-\widehat{D}_{t}$,

$$
\widehat{D}_{t}=\frac{1}{(T-1) h_{1}^{d}} \sum_{s \neq t} K_{1}\left(\frac{z_{s}-z_{t}}{h_{1}}\right) D_{s} / \hat{f}\left(z_{t}\right)
$$

with $\hat{f}\left(z_{t}\right)$ being the kernel density estimator of $f\left(z_{t}\right)$ and $K_{1}(\cdot)$ being a product kernel function, and

$$
K_{t s}=K\left(\frac{\varpi_{s}-\varpi_{t}}{h}\right)=K\left(\frac{z_{s}-z_{t}}{h}, \frac{\omega_{s}-\omega_{t}}{h}\right)
$$

with $K(\cdot)$ being another product kernel function and both bandwidths $h$ and $h_{1}$ being the smoothing parameters.

Finally, under some assumptions, Cai et al. (2019) obtained the following asymptotic results, under $H_{0}$,

where

$$
\widetilde{S}_{T}=\frac{T h^{p / 2} S_{T}}{\sqrt{2} \widehat{\sigma}_{T}} \stackrel{d}{\longrightarrow} \mathcal{N}(0,1)
$$

$$
\widehat{\sigma}_{T}^{2}=\frac{1}{T(T-1) h^{p}} \sum_{t=1}^{T} \sum_{s \neq t}\left(\hat{\varepsilon}_{t} \hat{f}\left(z_{t}\right)\right)^{2} \cdot\left(\hat{\varepsilon}_{s} \hat{f}\left(z_{s}\right)\right)^{2} K_{t s}^{2}
$$


is a consistent estimator of $\sigma_{T}^{2}$ given by

$$
\sigma_{T}^{2}=E\left[f^{4}\left(z_{t}\right) f_{\varpi}\left(\varpi_{t}\right) \sigma^{4}\left(\varpi_{t}\right)\right] \cdot\left(\int K^{2}(u) d u\right)
$$

with $\sigma^{2}\left(\varpi_{t}\right)=E\left(\varepsilon_{t}^{2} \mid \varpi_{t}\right)$, and under $H_{1}, P\left(\widetilde{S}_{T}>Q_{T}\right) \rightarrow 1$ for any non-stochastic sequence $Q_{T}=o\left(T h^{p / 2}\right)$. Therefore, the decision rule is that $H_{0}$ is rejected at the significance level $\alpha_{0}$ if $\widetilde{S}_{T}>c$, where $c$ is the upper $\alpha_{0}$-percentile of the standard normal distribution. Indeed, this test procedure can be used in practice. The reader is referred to the paper by Cai et al. (2019) for real examples.

\subsection{RDD in Time Series}

Regression discontinuity design (RDD) is another popular framework to identify treatment effects; see, for example, Thistlethwaite and Campbell (1960), Klaauw (2008), Lee and Card (2006), Lee (2008), Angrist and Lavy (1999), Ludwig and Miller (2007), and Hahn, Todd, and Klaauw (2001) for details. Kuersteiner et al. (2018) extended the RDD approach to a timeseries environment. Let $D_{t}$ follow a fixed rule $D_{t}=1\left\{R_{t}>c\right\}$, where the running variable $R_{t}$ is a continuous, nonrandom function of $z_{t}$ such that $R_{t}=g\left(z_{t}\right)$ for some function $g(\cdot), z_{t}$ is a covariate, and $c$ is a known threshold. Assume the potential outcome functions are continuous at the cutoff point $c$, i.e., $\eta_{t, l}$ in $Y_{t+l}=F_{t, l}\left(z_{t}, D_{t}, \eta_{t, l}\right)$ satisfying $E\left[\eta_{t, l} \mid R_{t}=c\right]$ is a.s. continuous at $c$. Then, around the critical point $c$, the treatment effect is given by:

$$
\theta_{l}(c)=\lim _{\Delta \downarrow 0} E\left[Y_{t+l} \mid R_{t}=c+\Delta\right]-\lim _{\Delta \uparrow 0} E\left[Y_{t+l} \mid R_{t}=c-\Delta\right] .
$$

The estimator $\hat{\theta}_{l}(c)$ is obtained by solving the problem:

$$
\left(\hat{a}, \hat{b}, \hat{\gamma}, \hat{\theta}_{l}(c)\right)=\arg \min _{a, b, \gamma, \theta_{l}} \sum_{t=2}^{T-l}\left(y_{t+l}-a_{l}-b_{l}\left(R_{t}-c\right)-\theta_{l} D_{t}-\gamma_{l}\left(R_{t}-c\right) D_{t}\right)^{2} K\left(\frac{R_{t}-c}{h}\right),
$$

where $K(\cdot)$ is a kernel function and $\mathrm{h}$ is a bandwidth. To illustrate the importance of RDD in practice, we use the example in Kuersteiner et al. (2018) to investigate how to use RDD in macroeconomic policy evaluation.

Example 3.1. In October 1999, the Central Bank of Colombia (CBoC henceforth) adopted an inflation-targeting regime with a floating exchange rate. Meanwhile, to control the volatility of exchange rate, $C B o C$ carried out rule-based currency interventions. The mechanics of rule-based interventions were as follows: at the close of any business day, whenever the average exchange rate for the entire day $e_{t}$, appreciated or depreciated (vis-a-vis its last 20-day moving average $\left.\bar{e}_{t}\right)$ at a rate faster than a cutoff $r_{t}$, the rule would be triggered, and call or put options on pesos would be issued. Options expired one month after the issued day, and could be exercised on days that the rule was triggered. There are two necessary conditions for $\mathrm{CBoC}$ to issue options:

(1) The appreciating or depreciating rate of $e_{t}$ exceeds the cutoff $r_{t}$;

(2) There are no outstanding options from a previous auction.

Thus, the running variable can be defined as:

$$
C_{t}=\frac{1}{r_{t}} \frac{e_{t}-\bar{e}_{t}}{\bar{e}_{t}}\left(1-O C_{t}\right), \quad \text { and } \quad P_{t}=\frac{1}{r_{t}} \frac{e_{t}-\bar{e}_{t}}{\bar{e}_{t}}\left(1-O P_{t}\right) \text {, }
$$


where $C_{t}$ is the running variable for issue call options on pesos, $P_{t}$ is the running variable for issue put options on pesos, and $O C_{t}\left(O P_{t}\right)$ is a dummy variable denoting whether call (put) options from a previous auction remain outstanding at date $t$. The policy intervention dummies $\left(D_{C, t}, D_{P, t}\right)$ are defined as, $D_{C, t}=\mathbf{1}\left\{C_{t} \geq 1\right\}$ with $R_{t}=C_{t}$ and $c=1$, and $D_{P, t}=\mathbf{1}\left\{P_{t} \leq-1\right\}$ with $R_{t}=P_{t}$ and $c=-1$. During the time period from January 2002 to February 2012, the rule was trigged 231 times, 38 auctions were issued, and options were exercised in 75 cases. The aim is to evaluate the dynamic treatment effects of the rule-based interventions on exchange rates. Here, $Y_{t}$ is change in log exchange rate, i.e. $Y_{t}=\log e_{t+l}-\log e_{t}$, where $l>0$.

In this example, the authors used the RDD method to measure the dynamic treatment effects. Consider the put options, for example, by assuming that $E\left[\eta_{t, l} \mid P_{t}=-1\right]$ is continuous, where $\eta_{t, l}$ is the unobservable shocks of $Y_{t, l}$ as defined above. Then, if $P_{t}$ is very close to -1 , based on the facts that traders can neither manipulate the running variable nor predict with certainty whether the rule is triggered or not, they conclude that any movement in $P_{t}$ at this point is as good as random noise. While the small movement of $P_{t}$ has a small effect on the average $\eta_{t, j}$, the movement can move $D_{P, t}$ from 0 to 1 or vice versa. Therefore, the movement when $P_{t}$ is local to the cutoff is as good as if we could randomly change $D_{P, t}$, and we obtain a local random assignment mechanism. Thus, the local dynamic treatment effect of put options can be estimated by:

$$
\theta_{l}=\lim _{p \downarrow(-1)} E\left[Y_{t+l} \mid P_{t}=p\right]-\lim _{p \uparrow(-1)} E\left[Y_{t+l} \mid P_{t}=p\right] .
$$

Define the dynamic treatment effect for horizons $1, \cdots L$ as:

$$
\theta=\left(\theta_{1}, \cdots, \theta_{L}\right)^{\prime} \text {. }
$$

Then, $\hat{\theta}$ can be obtained by local linear method:

$$
(\hat{a}, \hat{b}, \hat{\gamma}, \hat{\theta})=\arg \min _{a, b, \gamma, \theta} \sum_{l=1}^{L} \sum_{t=2}^{T-L}\left(y_{t+l}-a_{l}-b_{l}\left(P_{t}+1\right)-\theta_{l} D_{P, t}-\gamma_{l}\left(P_{t}+1\right) D_{P, t}\right)^{2} K\left(\frac{P_{t}+1}{h}\right)
$$

with $c=-1$. $\hat{\theta}$ obtained by RDD method can be cast in both a local nonlinear impulse response function framework and a potential outcome framework, which again illustrates the link between dynamic treatment effects defined by potential outcomes and classic macroeconomic model. Consider a perturbation $\epsilon(\delta)$ of $z_{t}$ such that the function of running variable $R_{t}=g\left(z_{t}\right)$ satisfying $g\left(z_{t}+\epsilon\right)-g\left(z_{t}\right)=\delta$ with $\delta>0$. For $\delta$ fixed and $\epsilon=\epsilon(\delta)$, the nonlinear impulse response function of $y_{t+j}$ is:

$$
\theta_{l}\left(\epsilon, z_{t}\right)=F_{t, l}\left(D\left(z_{t}+\epsilon\right), z_{t}+\epsilon\right)-F_{t, l}\left(D\left(z_{t}\right), z_{t}\right) .
$$

Since $F_{t, l}(\cdot)$ is continuous, far away the point $R_{t}=c$, we have $\lim _{\delta \rightarrow 0} \theta_{l}\left(\epsilon(\delta), z_{t}\right)=0$, and around the point $R_{t}=c$, we have $\theta_{l}^{R D D}\left(z_{t}\right)=F_{t, l}\left(1, z_{t}\right)-F_{t, l}\left(0, z_{t}\right)$. According to the definition of dynamic potential outcomes given by Angrist and Kuersteiner (2011) and Angrist et al. (2018), we know that $F_{t, l}\left(1, z_{t}\right)=Y_{t, l}(1)$, and $F_{t, l}\left(0, z_{t}\right)=Y_{t, l}(0)$. Therefore, the local nonlinear impulse response function is simply the treatment effect defined by potential outcomes.

From the equation $\theta_{l}^{R D D}\left(z_{t}\right)=F_{t, l}\left(1, z_{t}\right)-F_{t, l}\left(0, z_{t}\right)$, we can check the different conditions among traditional impulse response function, selection on observables, and RDD. Write the 
observed outcome $Y_{t+l}$ as:

$$
Y_{t+l}=F_{t, l}\left(0, z_{t}\right)+\theta_{l}^{R D D}\left(z_{t}\right) D_{t} .
$$

When assuming $F_{t, l}\left(0, z_{t}\right)$ is linear in $z_{t}, \theta_{l}^{R D D}\left(z_{t}\right)$ is a constant, and $E\left[D_{t} \mid z_{t}, F_{t, l}(\cdot)\right]=E\left[D_{t} \mid z_{t}\right]$ (selection on observables), (11) is a linear regression function, and $\theta_{l}^{R D D}\left(z_{t}\right)$ is the traditional

impulse response coefficient. Without assuming that $F_{t, l}\left(0, z_{t}\right)$ is linear in $z_{t}$ and $\theta_{l}^{R D D}\left(z_{t}\right)$ is a constant, the framework of selection on observables introduced in Section 3.3.1 can be applied. When the assumption of selection on observables fails, if a policy is implemented based on a clear rule, we can use the RDD method to estimate the dynamic treatment effects under the assumption that the potential outcomes function $F_{t, l}\left(z_{t}, D_{t}, \eta_{t, l}\right)$ is a.s. continuous at $c$.

\section{$\S 4$ Evaluation Methods with Multiple Time Series Data}

In this section, we survey the existing approaches for estimation of macroeconomic policy effects with multiple time series data. We first discuss synthetic control method (SCM) and then examine the panel data approach proposed by Hsiao et al. (2012). In this setting, we have observations of multiple periods on multiple units, indexed by $i=1, \cdots, N, t=1, \cdots, T$. At time $T=1, \cdots T_{1}\left(1 \leq T_{1}<T\right)$, there are no units exposed to the policy intervention. From time $T_{1}+1$ to $\mathrm{T}$, only one unit is uninterruptedly exposed to the policy, while others are not. Without loss of generality, suppose that the first unit $i=1$ is treated.

\subsection{Synthetic Control Method}

A way to assess treatment effects for the multiple time series setting is the synthetic control method (SCM), which is proposed by Abadie and Gardeazabal (2003), and Abadie, Diamond and Hainmueller (2010). The main idea behind SCM is using a linear combination of all control units to form an artificial control unit that is more similar to the treatment unit in the preintervention periods than to any of control units on their own. The counterfactual outcomes after the implementation periods for the treatment unit is estimated as a weighted average of the control units in the same periods. The SCM method is illustrated in detail using the example in Abadie and Gardeazabal (2003).

Example 4.1. Assume there are $N$ regions, $i=1, \cdots N$. The first unit $(i=1)$ is Basque Country, and the others are other Spanish regions. The regions are observed at periods from 1955 to 1997 , i.e., $t=1, \cdots T, t=1$ denotes the year 1955, and $t=T$ denotes the year 1997 . From year $T_{1}+1\left(T_{1}=1967\right)$, Euskadi Ta Askatasuna (ETA), a Basque terrorist organization, claimed its first victim in Basque Country and continued afterwards. ETA's terrorist activity was no more than two victims per year before 1973, increased to almost 16 victims per year on average in the period 1974-1977, and peaked to a total of 235 victims in the years of 1978-1980, after which it decreased gradually. In September 1998, ETA declared a total and indefinite cease fire. Since ETA's violent activity has been concentrated in the Basque Country, other areas are deemed to not require such treatment. Let $D_{i t}$ denote a binary treatment indicator, Thus, we have the data $\left\{\left(D_{i t}, Y_{i t}, \mathbf{X}_{i}\right)\right\}$, where $D_{i t}=0$ for $i=1 \cdots N$ and $t=1, \cdots, T_{1}, D_{1 t}=1$ for 
$t=T_{1}+1, \cdots, T$, and $D_{i t}=0$ for $i=2, \cdots, N$ and $t=T_{1}+1, \cdots, T$, and $Y_{i t}$ is per capita $G D P$ for region $i$ at time $t, \mathbf{X}_{i}$ is a vector of economic growth predictors for region $i$ with the dimension of $K$ (not affected by the intervention).

The main target of the analysis in Abadie and Gardeazabal (2003) is to evaluate the impact that terrorism has had on economic growth for the Basque Country. Since there is no intervention before $T_{1}$, it is clear that $Y_{i t}=Y_{i t}(0)$ for $i=1, \cdots, N, t=1, \cdots, T_{1}$. After $T_{1}$, only the first unit uninterruptedly receives the treatment. Then, $Y_{1 t}=Y_{1 t}(1)$ for $t=T_{1}+1, \cdots, T$ and $Y_{i t}=Y_{i t}(0)$ for $i=2, \cdots, N$ and $t=T_{1}+1, \cdots, T$. To recover $Y_{1 t}(0)$ for periods $t=T_{1}+1, \cdots, T$, Abadie and Gardeazabal (2003) conceptualized a weighted average of other Spanish regions as a "synthetic" Basque Country without terrorism; that is,

$$
Y_{1 t}(0)=\sum_{i=2}^{N} w_{i} \cdot Y_{i t}, \quad t=T_{1}+1, \cdots, T,
$$

where $w_{i}(i=2, \cdots, N)$ represents the weight of region $i$ in the synthetic Basque Country, $w_{i} \geq 0$, and $\sum_{i=2}^{N} w_{i}=1$. Let $W=\left(w_{2}, \cdots, w_{N}\right)^{\prime}$. Then, each different value of $W$ represents a different synthetic Basque Country. Theoretically, $W$ is obtained by minimizing

$$
\left(Z_{1}-Z_{0} W\right)^{\prime} V\left(Z_{1}-Z_{0} W\right)^{\prime}
$$

with $w_{i} \geq 0$ and $\sum_{i=2}^{N} w_{i}=1$, subject to the following two constrains:

$$
Y_{1 t}=\sum_{i=2}^{N} w_{i} Y_{i t}, t=1, \cdots T_{1},
$$

and

$$
\mathbf{X}_{1}=\sum_{i=2}^{N} w_{i} \mathbf{X}_{i}
$$

Here, $Z_{1}=\left(Y_{11}, \cdots, Y_{1 T_{0}}, \mathbf{X}_{11}, \cdots, \mathbf{X}_{1 K}\right)^{\prime}$ is a $\left(T_{1}+K\right) \times 1$ vector of outcomes before $T_{1}$ and average values of $\mathrm{K}$ covariates from period 1 to $T_{1}$ for Basque Country, and $Z_{0}$ is a $\left(T_{1}+K\right) \times$ $(N-1)$ matrix of the same values of other N-1 regions. Further, $V$ is a $\left(T_{1}+K\right) \times\left(T_{1}+K\right)$ positive-definite matrix, with the diagonal elements $v_{i i}$ reflecting the relative importance of the different variables in determining $Y$. The choice of $V$ could be subjective.

After obtaining the optimal weights $w_{i}^{*}$, the treatment effect can be estimated by:

$$
\hat{\Delta}_{1 t}=Y_{1 t}-\sum_{i=2}^{N} w_{i}^{*} Y_{i t} .
$$

In practice, it is almost impossible to find the optimal weights $w_{i}^{*}$ to satisfy both (13) and (14) for the given data. Thus, the weight $w_{i}$ is often selected based only on (12). As for SCM method, it can be regarded as a generalization of the differnce-in-differences (DID) model. A standard SCM model is supposed as:

$$
Y_{i t}(0)=\delta_{t}+b_{i}^{\prime} f_{t}+\beta_{t}^{\prime} \mathbf{X}_{i}+\epsilon_{i t}, \quad \text { and } \quad Y_{i t}(1)=Y_{i t}(0)+\Delta_{i t} D_{i t},
$$

where $\delta_{t}$ is an unknown common factor with constant factor loadings across units, $f_{t}$ is a vector of unknown common factors with varying factor loadings $b_{i}, \mathbf{X}_{i}$ is a vector of covariates (independent from intervention and time-invariant), $\beta_{t}$ is a vector of unknown parameters with possible time-varying, and $\epsilon_{i t}$ is unobserved shock with zero mean for all unit $i$. If we impose 
that $f_{t}$ is constant for all $\mathrm{t}$ in $\mathrm{SCM}$ :

$$
Y_{i t}(0)=\delta_{t}+f b_{i}+\beta_{t}^{\prime} \mathbf{X}_{i}+\epsilon_{i t}
$$

the DID model is obtained with $b_{i}=g_{i}$, where $g_{i}$ is the group unit i belonged to, and $g_{i} \in$ $\{0,1, \cdots, G\}$. Thus, the SCM model allows for the time-varying effects of unobserved common factors, but the DID model restricts the effects of these unobservable common factors to be constant in time.

\subsection{HCW Method}

Hsiao et al. (2012) proposed a more flexible and more simple-to-implement panel data methodology to analyze treatment effects. This method exploited the correlations among cross sectional units to construct the counterfactuals. Hsiao et al. (2012) argued the cross-sectional dependence is due to the presence of some unobserved common factors. Based on this point, they developed a straightforward way to construct counterfactuals with observed data. In this section, we discuss the basic model and some extensions of their approach.

\subsubsection{Model Setup}

The setting is the same as that in SCM. That is, $Y_{1 t}=Y_{1 t}(0)$ for $t=1, \cdots, T_{1}, Y_{1 t}=Y_{1 t}(1)$, for $t=T_{1}+1, \cdots, T$, and $Y_{i t}=Y_{i t}(0)$ for $i=2, \cdots, N$ and $t=1, \cdots, T$. To recover $Y_{1 t}(0)$ for periods $T_{1}+1, \cdots, T$, the HCW method assumes that $Y_{i}(0)$ has the following factor model:

$$
Y_{i t}(0)=\alpha_{i}+b_{i}^{\prime} f_{t}+\epsilon_{i t}, \quad i=1, \cdots N ; \quad t=1, \cdots T,
$$

where $\alpha_{i}$ is the fixed individual-specific effect, $f_{t}$ is a $K \times 1$ vector of unobserved common factors that vary over time, $b_{i}$ is a $K \times 1$ vector of factor loadings that vary across $i, \epsilon_{i}$ is the unobservable random idiosyncratic component with $E\left[\epsilon_{i}\right]=0$, and $E\left[\epsilon_{i} \epsilon_{j}\right]=0$ for $i \neq j$. The $\mathrm{HCW}$ model can be expressed in terms of matrix form as:

$$
Y_{t}(0)=\alpha+B f_{t}+\epsilon_{t},
$$

where $Y_{t}(0)=\left(Y_{1 t}(0), \ldots, Y_{N t}(0)\right)^{\prime}, \alpha=\left(\alpha_{1}, \ldots \alpha_{N}\right)^{\prime}, \epsilon_{t}=\left(\epsilon_{1 t}, \ldots, \epsilon_{N t}\right)^{\prime}$, and B is the $N \times K$ factor loading matrix $B=\left(b_{1}, \ldots, b_{N}\right)^{\prime}$. To estimate $Y_{1 t}(0)$ for periods $T_{1}+1, \cdots, T$, it is needed to assume that

$$
E\left[\epsilon_{j s} \mid D_{i t}\right]=0 \text { for } j \neq i
$$

The assumption in (16) means that other units are not affected by the policy implemented to the treatment unit.

Let $v=\left(1,-\gamma^{\prime}\right)^{\prime}$ and $\gamma=\left(\gamma_{2}, \cdots \gamma_{N}\right)^{\prime}$, such that $v^{\prime} B=0$, i.e., $v \in \mathcal{N}(B)$, where $\mathcal{N}(B)$ is the null space of $B$. Then,

$$
v^{\prime} Y_{t}(0)=v^{\prime} \alpha+v^{\prime} \epsilon_{t}
$$

Rearranging, we obtain

$$
Y_{1 t}(0)=\gamma_{1}+\gamma^{\prime} \tilde{Y}_{t}+\epsilon_{1 t}^{*}
$$

where $\gamma_{1}=v^{\prime} \alpha, \tilde{Y}_{t}=\left(Y_{2 t}, \cdots, Y_{N t}\right)^{\prime}, \epsilon_{1 t}^{*}=v^{\prime} \epsilon_{t}=\epsilon_{1 t}-\gamma^{\prime} \tilde{\epsilon}_{t}$, and $\tilde{\epsilon}_{t}=\left(\epsilon_{2 t}, \cdots, \epsilon_{N t}\right)^{\prime}$. Since $\tilde{Y}_{t}$ 
is correlated with $\epsilon_{1 t}^{*}$, we decompose $\epsilon_{1 t}^{*}=E\left(\epsilon_{1 t}^{*} \mid \tilde{Y}_{t}\right)+\eta_{1 t}$ and $E\left[\eta_{1 t} \mid \tilde{Y}_{t}\right]=0$. Then,

$$
Y_{1 t}(0)=\gamma_{1}+\gamma^{\prime} \tilde{Y}_{t}+E\left(\epsilon_{1 t}^{*} \mid \tilde{Y}_{t}\right)+\eta_{1 t}
$$

Furthermore, it is assumed that

$$
E\left(\epsilon_{1 t}^{*} \mid \tilde{Y}_{t}\right)=a_{0}+b_{0}^{\prime} \tilde{Y}_{t}
$$

Then,

$$
Y_{1 t}(0)=a+c^{\prime} \tilde{Y}_{t}+\eta_{1 t}, \quad t=1, \cdots, T,
$$

where $a=a_{0}+\gamma_{1}$, and $c=\gamma+b_{0}$, which means that we can use observations of $Y_{2 t}, \cdots, Y_{N t}$ to predict $Y_{1 t}(0)$. Later, Li and Bell (2017) showed that without the assumption in $(17)$, (18) still holds. By minimizing the following equation:

$$
\frac{1}{T_{1}}\left(Y_{1}(0)-a^{\prime} \mathbf{e}-c^{\prime} Y_{-1}\right) A\left(Y_{1}(0)-a^{\prime} \mathbf{e}-c^{\prime} Y_{-1}\right)
$$

we can obtain a consistent ordinary least squares (OLS) estimator of $a$ and $c$. Here, $Y_{1}(0)=$ $\left(Y_{11}, \cdots, Y_{1 T_{1}}\right)^{\prime}$, e is a $T_{1} \times 1$ vector of 1 's, $Y_{-1}$ is a $T_{1} \times(N-1)$ matrix of $T_{1}$ time series observations of $\tilde{Y}_{t}$, A is a $T_{1} \times T_{1}$ positive definite matrix. So, $Y_{1 t}(0)$ for periods $t=T_{1}+1, \cdots, T$ is:

$$
\hat{Y}_{1 t}(0)=\hat{a}+\hat{c}^{\prime} \tilde{Y}_{t}, \quad t=T_{1}+1, \cdots, T .
$$

Thus, the treatment effect for unit 1 is:

$$
\hat{\Delta}_{1 t}=Y_{1 t}-\hat{Y}_{1 t}(0), \quad t=T_{1}+1, \cdots, T .
$$

The standard deviation of $\hat{Y}_{1 t}(0)$, denoted by $\sigma_{Y_{1 t}(0)}$, can be calculated by the standard formula for standard deviation. For example, when $\eta_{1 t}$ is iid, then

$$
\sigma_{Y_{1 t}(0)}^{2}=\sigma_{\eta_{1}}^{2}\left[1+\left(1, \tilde{Y}_{t}^{\prime}\right)\left(Y_{-1}^{\prime} Y_{-1}\right)^{-1}\left(1, \tilde{Y}_{t}^{\prime}\right)\right]
$$

The confidence interval of $\Delta_{1 t}$ is correspondingly as $\hat{\Delta}_{1 t} \pm c \sigma_{Y_{1 t}(0)}$. Furthermore, suppose $\Delta_{1 t}$ follows an autoregressive moving average model (ARMA):

$$
a(L) \Delta_{1 t}=\mu+\theta(L) \eta_{t},
$$

where $L$ is the lag operator, $\eta_{t}$ is white noise, and the roots of $\theta(L)=0$ lie outside the unit circle. If all roots of $a(L)=0$ lie outside the unit circle, then the long-term treatment effect is

$$
\Delta_{1}=a(L)^{-1} \mu=\mu^{*},
$$

and $\Delta_{1}$ can be estimated by taking the simple average of the treatment effect:

$$
\hat{\Delta}_{1}=\frac{1}{T_{2}} \sum_{t=T_{1}+1}^{T} \hat{\Delta}_{1 t},
$$

where $T_{2}=T-T_{1}$. Indeed, Li and Bell (2017) derived the consistency and asymptotic distribution of $\hat{\Delta}_{1}$ by showing that

$$
\hat{\Delta}_{1}-\Delta_{1}=O_{p}\left(T_{1}^{-1 / 2}+T_{2}^{-1 / 2}\right)
$$

and

$$
\sqrt{T_{2}}\left(\hat{\Delta}_{1}-\Delta_{1}\right) \stackrel{d}{\rightarrow} N(0, \Sigma)
$$

where $\Sigma=\Sigma_{1}+\Sigma_{2}, \Sigma_{1}=\eta E\left(\chi_{t}\right)^{\prime} V E\left(\chi_{t}\right), \eta=\lim _{T_{1}, T_{2} \rightarrow \infty} T_{2} / T_{1}, \chi_{t}=\left(1, \tilde{Y}_{t}^{\prime}\right)^{\prime}, V=$ $\lim _{T_{1}, T_{2} \rightarrow \infty} \operatorname{Var}\left(\sqrt{T_{1}} \hat{\beta}\right), \hat{\beta}=\left(\hat{a}, \hat{c}^{\prime}\right)^{\prime}$, and $\Sigma_{2}=\lim _{T_{1}, T_{2} \rightarrow \infty} \operatorname{Var}\left(T_{2}^{-1 / 2} \sum_{t=T_{1}+1}^{T}\left(\Delta_{1 t}-E\left(\Delta_{1 t}\right)+\eta_{1 s}\right)\right)$. Obviously, the asymptotic normality in (19) can be used for making inferences for $\Delta_{1}$ provided 
that a consistent estimate of $\Sigma$ in (19) is available.

The above is the main idea of the HCW method. By using this method, Hsiao et al. (2012) assessed the effect of political and economic integration of Hong Kong with mainland China after 1997 on Hong Kong's economic growth.

\subsubsection{Selection of Control Units}

In real applications, the question how to select control units rises. It is well known that when $\tilde{Y}_{t}$ is used to predict $Y_{1 t}(0)$, it is not true that the number of $\tilde{Y}_{t}$ should be as many as possible. As more units are used, the variance should increase. To balance the within-sample fit and postsample prediction error, two criteria must be met to select control units. One is that control units must display strong correlations with the treatment unit based on the pretreatment data. The other criterion is that control units should be independent of the treatment.

Hsiao et al. (2012) proposed a two-step way to choose the most strong correlated predictors. First, assume we can use $j$ units to predict $Y_{1 t}(0), j=1, \cdots, N-1$ and then use $R^{2}$ or likelihood values to select the best predictor for $Y_{1 t}(0)$, denoted by $M(j)^{*}$. Second, choose the best $M(m)^{*}$ from $M(1)^{*}, M(2)^{*}, \cdots, M(N-1)^{*}$ in terms of a model selection criterion.

The essence of the procedure proposed by Hsiao et al. (2012) is to minimize the Akaike information criterion (AIC) or its extension (corrected version of AIC, AICC) or the Baysian information criterion (BIC). However, AIC and AICC are asymptotically inconsistent (Shao, 1993, 1996) because neither the probability of selecting the optimal model nor the probability of selecting the model with the best predictive ability converges to 1 as $T \rightarrow \infty$. To overcome this problem, Du and Zhang (2015) suggested using a leave- $n_{v}$-out cross-validation criterion $\left(\mathrm{CV}\left(n_{v}\right)\right)$ to choose the optimal control units, which has the consistent property. The $\mathrm{CV}\left(n_{v}\right)$ method first chooses the data from cross-sectional dimension, the chosen subset of $\{2, \cdots, N\}$ is denoted by $S$, and the components in $S$ are $\tilde{Y}_{S t}$. Then, split the data into two parts from the time dimension. That is, $\left\{Y_{1 t}, \tilde{Y}_{S t}^{\prime}\right\}_{t=1}^{T_{1}}$ is split into $\left.\left\{\left(Y_{1 k}, \tilde{Y}_{S k}^{\prime}\right), k \in \kappa\right\},\left\{Y_{1 k}, \tilde{Y}_{S k}^{\prime}\right), k \in \kappa^{c}\right\}$, where $\kappa$ is a subset of $\left\{1, \cdots, T_{1}\right\}$ with $n_{v}$ elements and $\kappa^{c}$ is its complement. After that, regression $Y_{1 k}$ on constant and $\tilde{Y}_{S k}$ using the data indexed by $\kappa^{c}$, and obtain the estimation of $\hat{a}_{S, \kappa^{c}}$ and $\hat{c}_{S, \kappa^{c}}$. Calculate the average squared prediction error using the data indexed by $\kappa, \frac{1}{n_{v}} \sum_{k \in \kappa}\left(Y_{1 k}-\hat{a}_{S, \kappa^{c}}-\hat{c}_{S, \kappa^{c}}^{\prime} \tilde{Y}_{S k}\right)^{2}$. There are $C_{T_{1}}^{n_{v}}$ ways to divide $1,2, \cdots T_{1}$ into $\kappa$ and $\kappa^{c}$. Therefore, $M$ of them are randomly drawn, as $\left\{\kappa_{j}, \kappa_{j}^{c}\right\}_{j=1}^{M}$, and the objective function is defined as

$$
\frac{1}{n_{v} M} \sum_{j=1}^{M} \sum_{k \in \kappa_{j}}\left(Y_{1 k}-\hat{a}_{S, \kappa_{j}^{c}}-\hat{c}_{S, \kappa_{j}^{c}}^{\prime} \tilde{Y}_{S k}\right)^{2} .
$$

From all the possible subsets of $\{2, \cdots N\}$, Du and Zhang (2015) chose $S^{*}$ to minimize the objective function defined in (20). They showed that the $\mathrm{CV}\left(n_{v}\right)$ method can produce the consistent estimate and further, illustrated that the $\mathrm{C} V\left(n_{v}\right)$ method gives smaller out-of-sample prediction results than AIC and AICC via simulations.

Instead of using some classical model selection approaches, Li and Bell (2017) proposed using the least absolute shrinkage and selection operator (Lasso) method, a modern model 
selection method, to select control units based on the following three reasons. First, when the number of control units is larger than the number of pretreatment time periods $\left(N>T_{1}\right)$, the conventional model selection methods would break down. The second reason is that the Lasso method is computationally efficient. The third reason is that simulations show that the Lasso method has smaller out-of-sample prediction errors than AIC, AICC, BIC, and CV $\left(n_{v}\right)$. The objective function of the Lasso method is

$$
\sum_{t=1}^{T_{1}}\left(Y_{1 t}-\chi_{t}^{\prime} \beta\right)^{2}+\lambda \sum_{j=1}^{N}\left|\beta_{j}\right|,
$$

where $\chi_{t}=\left(1, \tilde{Y}_{t}^{\prime}\right)^{\prime}, \beta=\left(\hat{a}, \hat{c}^{\prime}\right)^{\prime}$, and $\lambda$ is a tuning parameter. The larger $\lambda$ is, the more penalty is imposed on nonzero $\beta_{j}$. To obtain a Lasso estimator of $\beta$, a value of $\lambda$ must first be selected. Li and Bell (2017) adopted the leave-one-out cross validation method to select $\lambda$ over a discrete set $\Lambda_{L}=\left\{\lambda_{1}, \lambda_{2}, \cdots, \lambda_{L}\right\}$. For each $\lambda \in \Lambda_{L}$, and each $t=1, \cdots, T_{1}$, by minimizing the following leave-one-out objective function:

$$
\sum_{k=1, k \neq t}^{T_{1}}\left(Y_{1 k}-\chi_{k}^{\prime} \beta\right)^{2}+\lambda \sum_{l=1}^{N}\left|\beta_{l}\right|,
$$

we get the leave-one-out estimator of $\beta_{-t, \lambda}$. Then, we compute the $\operatorname{CV}(\lambda)$,

$$
\mathrm{CV}(\lambda)=\frac{1}{T_{1}} \sum_{t=1}^{T_{1}}\left(Y_{1 t}-\chi_{t}^{\prime} \beta_{-t, \lambda}\right)^{2}
$$

Finally, we choose $\lambda$ to minimize $\operatorname{CV}(\lambda)$.

\subsubsection{Semiparametric Model}

Ouyang and Peng (2015) relaxed the linear conditional mean assumption in (17) and extended the HCW model to a semiparametric setting. Without imposing condition in (17), (18) becomes:

$$
Y_{1 t}(0)=g\left(\tilde{Y}_{t}\right)+\eta_{1 t}
$$

where $g\left(\tilde{Y}_{t}\right)=\gamma_{1}+\gamma^{\prime} \tilde{Y}_{t}+E\left(\epsilon_{1 t}^{*} \mid \tilde{Y}_{t}\right)$, and $g\left(\tilde{Y}_{t}\right)$ can be estimated using a nonparametric method, such as the local constant kernel method, using data before $T_{1}$ :

$$
\hat{g}\left(\tilde{Y}_{t}\right)=\frac{\sum_{s=1}^{T_{1}} Y_{1 s} K_{n h}}{\sum_{s=1}^{T_{1}} K_{n h}}, \quad t=T_{1}+1, \cdots, T,
$$

where $K_{n h}=\Pi_{j=2}^{N} k\left(\left(\tilde{Y}_{s, j}-\tilde{Y}_{t, j}\right) / h_{j}\right)$ is the product kernel function, and $h_{j}$ is the bandwidth associated with covariate $Y_{t, j}$ for $j=2, \cdots, N$. The average treatment effect can then be estimated:

$$
\hat{\Delta}_{1, N P}=\frac{1}{T_{1}} \sum_{t=T_{1}+1}^{T}\left(Y_{1 t}-\hat{g}\left(\tilde{Y}_{t}\right)\right) .
$$

To avoid the so-called "curse of dimensionality" problem for large $N, g\left(\tilde{Y}_{t}\right)$ can be estimated semi-parametrically:

$$
g\left(\tilde{Y}_{t}\right)=\beta^{\prime} z_{1 t}+h\left(z_{2 t}\right),
$$

where $z_{1 t} \cup z_{2 t}=\tilde{Y}_{t}$, and $z_{1 t} \cap z_{2 t}=\emptyset$. However, the authors did not discuss how to choose $z_{1 t}$ 
and $z_{2 t}$. If $q$, the dimension of $z_{2 t}$ is low, the "curse of dimensionality" problem can be greatly alleviated. $\hat{\beta}$ and $\hat{h}$ can be estimated by the profile least squares method using the pretreatment data. First, we treat $\beta$ as if it were known and estimate $h\left(z_{2 t}\right)$ by

$$
\tilde{h}\left(z_{2 t}\right)=T_{1}^{-1} \sum_{s=1}^{T_{1}}\left(Y_{1 s}-\beta^{\prime} z_{1 t}\right) K_{s h} / \hat{f}_{t}^{*}=B_{1 t}-B_{2 t}^{\prime} \beta,
$$

where $B_{1 t}=T_{1}^{-1} \sum_{s=1}^{T_{1}} Y_{1 s} K_{s h} / \hat{f}_{t}^{*}, B_{2 t}=T_{1}^{-1} \sum_{s=1}^{T_{1}} z_{1 t} K_{s h} / \hat{f}_{t}^{*}, \hat{f}_{t}^{*}=T_{1}^{-1} \sum_{s=1}^{T_{1}} K_{s h}$, and $K_{s h}=\Pi_{l=1}^{q} h_{l}^{-1} k\left(\left(z_{2 s, l}-z_{2 t, l}\right) / h_{l}\right)$. Clearly, $\tilde{h}\left(z_{2 t}\right)$ is not feasible, because $\beta$ is unknown. Replacing $h\left(z_{2 t}\right)$ in $(21)$ by $\tilde{h}\left(z_{2 t}\right)$, we obtain:

$$
Y_{1 t}-B_{1 t}=\left(z_{1 t}-B_{2 t}\right)^{\prime} \beta+\eta_{1 t} .
$$

Using OLS,

$$
\hat{\beta}=\left[\sum_{t=1}^{T_{1}}\left(z_{1 t}-B_{2 t}\right)\left(z_{1 t}-B_{2 t}\right)^{\prime}\right]^{-1} \sum_{t=1}^{T_{1}}\left(z_{1 t}-B_{2 t}\right)\left(Y_{1 t}-B_{1 t}\right) .
$$

Plugging $\hat{\beta}$ into (22) yields

$$
\hat{h}\left(z_{2 t}\right)=T_{1}^{-1} \sum_{s=1}^{T_{1}}\left(Y_{1 s}-\hat{\beta}^{\prime} z_{1 t}\right) K_{s h} / \hat{f}_{t}^{*} .
$$

Finally, Ouyang and Peng (2015) applied the basic HCW and semiparametric HCW models to study the macroeconomic effect of the 2008 Chinese Economic Stimulus Program. They found that the fiscal stimulus plan increased the annual real GDP growth in China by approximately $3.2 \%$, but only temporarily. Next, we use this example to illustrate the practical usefulness of the basic and semiparametric HCW models in empirical applications.

Example 4.2. The 2008 Chinese Economic Stimulus Program was one of the most significant macroeconomic events of China over the past decades. Starting from November 9, 2008, the Chinese government implemented an economic stimulus package of four trillion RMB in order to minimize the impact of the global financial crisis. Here, $Y$ is the real GDP growth. The data include $N=39$ countries and $T=69$ periods $(T=1$ is the first quarter of 1996 , and $t=T$ is the first quarter of 2013), 51 for pretreatment periods and 18 for post-treatment periods. Set $T_{1}=51$ and assume China is the first unit. Then, $Y_{i t}=Y_{i t}(0)$ for $i=1, \cdots, N$ and $t=1, \cdots, T_{1}, Y_{1 t}=Y_{1 t}(1)$ for $t=T_{1}+1, \cdots, T$, and $Y_{i t}=Y_{i t}(0)$ for $i=2, \cdots, N$ and $t=T_{1}+1, \cdots, T$. To balance the within-sample fit and post-sample prediction error, we cannot directly use the other 38 counties to predict $Y_{1 t}(0)$ for post-treatment periods. We should choose the control counties using two criteria: strong correlation with China and exogenous to the treatment. They first constructed an exogenous measure that is the ratio of a country's trade volume with China in its own GDP. Countries with a ratio below $5 \%$ are chosen as candidates for control units. Accordingly, 31 counties satisfy the exogenous criterion. Then, using the two-step method proposed by Hsiao et al. (2012), the best predictors can be selected.

The basic HCW model and the corresponding semiparametric model used in their paper are as follows:

$$
Y_{1 t}(0)=\alpha+\gamma^{\prime} \tilde{Y}_{t}+\theta t+\beta^{\prime} \mathbf{X}_{t}+\eta_{1 t}
$$


and

$$
Y_{1 t}(0)=g\left(\tilde{Y}_{t}\right)+\theta t+\beta^{\prime} \mathbf{X}_{t}+\eta_{1 t}
$$

respectively, including a linear time trend, where $\mathbf{X}_{t}$ is a covariate vector to help with the fit of the data. By using OLS or profile least square, we can obtain $\hat{Y}_{1 t}(0)$, and the estimation of treatment effect for China is thus:

$$
\hat{\Delta}_{1 t}=Y_{1 t}-\hat{Y}_{1 t}(0), \quad t=52, \cdots, 69 .
$$

The average treatment effect is estimated by:

$$
\hat{\Delta}_{1}=\frac{1}{T-T_{1}} \sum_{t=T_{1}+1}^{T} \hat{\Delta}_{1 t} .
$$

Based on the analysis of real example using the aforementioned method, they found that the fiscal stimulus plan increased the annual real GDP growth in China by approximately $\hat{\Delta}_{1}=3.2 \%$.

\subsubsection{Disentangling the Effects of Multiple Treatments}

All the methods proposed above assume that there is only one treatment. However, in many cases, there may be several treatments working simultaneously. Fujiki and Hsiao (2015) extended the standard HCW to distinguish the effect of one treatment from the other when the units are exposed to "both" working treatments at the same time. We use their example of Great Hanshin-Awaji earthquake to introduce the idea behind this methodology.

Example 4.3. The Great Hanshin-Awaji earthquake took place on January 17, 1995. After that, the economic growth of the Kobe region declined dramatically. However, at approximately the same time, there was a fundamental structural change occurring around the disaster area. On the one hand, the port of Kobe was met by a challenge from other lower-cost ports in Asia such as Pusan, Hong Kong or Singapore. On the other hand, traditional industries, which are heavily concentrated in the disaster area, were challenged by globalization. For example, the chemical shoe industry, one of the most important local industries, had to compete with the cheaper shoes from China and the expensive shoes from Italy and France. How much the Great Hanshin-Awaji earthquake should account for this economic decline is the issue explored by Fujiki and Hsiao (2015).

Real GDP data from the 47 prefectures are available from 1955 to fiscal year 2009, i.e., we have observations of $N=47$ units in $T=54$ periods. $t=1$ is the fiscal year 1955, and $T_{1}$ is the fiscal year 1994. Before $T_{1}$, no unit received treatment. From $T_{1}+1$ onwards, the first unit, the Hyogo prefecture (the Kobe region) received two treatments $D_{1 t}$ and $D_{2 t}$, while the rest did not. $D_{1 t}$ is a binary indicator for the earthquake treatment, and $D_{2 t}$ is a binary indicator for the other treatment, such as structural economic change. Suppose the net effect of natural disaster is transitory. Then, from $T_{2}+1\left(T_{2}>T_{1}\right)$, the observed value no longer contains the earthquake effect. Here, $Y$ denotes the real GDP. Define $Y_{i t}=Y_{i t}(0,0)$ for $i=1, \cdots, N$ and $t=1, \cdots, T_{1}, Y_{1 t}=Y_{1 t}(1,1)$ for $t=T_{1}+1, \cdots, T_{2}, Y_{1 t}=Y_{1 t}(0,1)$ for $t=T_{2}+1, \cdots, T$, and $Y_{i t}=Y_{i t}(0,0)$ for $i=2, \cdots, N$ and $t=T_{1}+1, \cdots, T$, where $Y_{i t}(i, j)$ denotes the potential outcomes of $Y$, when $D_{1 t}=i$ and $D_{2 t}=j$ for $i=0$ and 1 , and $j=0$ and 1 . 
To measure the net impact of the earthquake on the economy of Kobe region, Fujiki and Hsiao (2015) first estimated $\hat{Y}_{1 t}(0,0)$ after period $T_{1}$ using the HCW method. That is,

$$
\hat{Y}_{1 t}(0,0)=E\left[Y_{1 t}(0,0) \mid \tilde{Y}_{t}\right]=\gamma_{1}+\gamma^{\prime} \tilde{Y}_{t}^{*}, \quad t=T_{1}+1, \cdots, T,
$$

where $\tilde{Y}_{t}^{*}$ is the chosen subset of $\tilde{Y}_{t}$ according to the two criteria to predict $Y_{1 t}(0,0)$. Using data from 1 to $T_{1}, \hat{\gamma}_{1}$ and $\hat{\gamma}$ are obtained and we have:

$$
\hat{Y}_{1 t}(0,0)=\hat{\gamma}_{1}+\hat{\gamma}^{\prime} \tilde{Y}_{t}^{*}, \quad t=T_{1}+1, \cdots, T .
$$

Since during periods $T_{1}+1 \cdots T_{2}, Y_{1 t}=Y_{1 t}(1,1)$, the treatment effects got by $Y_{1 t}-\hat{Y}_{1 t}(0,0)$ are the combined effects of earthquake and structural change. To isolate the net earthquake effects, Fujiki and Hsiao (2015) noticed the fact that only the treatment of structural change works after $T_{2}$. Using the same way, they constructed $\hat{Y}_{1 t}(0,1)$ before $T_{2}$,

$$
\hat{Y}_{1 t}(0,1)=E\left[Y_{1 t}(0,1) \mid \tilde{Y}_{t}\right]=\delta_{1}+\delta^{\prime} \tilde{Y}_{t}^{* *}, \quad t=1, \cdots, T_{2},
$$

where $\tilde{Y}_{t}^{* *}$ is the subset of $\tilde{Y}_{t}$ to predict $Y_{1 t}(0,1)$. Using data from $T_{2}+1$ to $T, \hat{\delta}_{1}$ and $\hat{\delta}$ are obtained and the estimation of potential outcome $Y_{1 t}(0,1)$ before $T_{2}$ is backcasted as:

$$
\hat{Y}_{1 t}(0,1)=\hat{\delta}_{1}+\hat{\delta}^{\prime} \tilde{Y}_{t}^{* *}, \quad t=1, \cdots, T_{2} .
$$

Then, the net earthquake effects from $T_{1}+1$ to $T_{2}$ are:

$$
\hat{\Delta}_{1 t}^{e}=Y_{1 t}-\hat{Y}_{1 t}(0,1), \quad t=T_{1}+1, \cdots, T_{2},
$$

and the net effects of structural change from $T_{1}+1$ to $T_{2}$ are estimated by:

$$
\hat{\Delta}_{1 t}^{s}=\hat{Y}_{1 t}(0,1)-\hat{Y}_{1 t}(0,0), \quad t=T_{1}+1, \cdots, T_{2} .
$$

That is the main idea of this methodology proposed by Fujiki and Hsiao (2015) to be used to separate one treatment from the other treatments. In the empirical study, the separation of one treatment from the other depends critically on the choice of $T_{2}$. Therefore, one should choose $T_{2}$ carefully and perform a robust check to ensure that the choice is reasonable. Finally, note that this approach by Fujiki and Hsiao (2015) can be easily generalized to the case with 3 or more than 3 treatments or interventions.

\subsubsection{Comparison of SCM and HCW}

As mentioned above, the HCW and SCM models are both applicable to the setting that only one unit is uninterruptedly exposed to the treatment after period $T_{1}$, while others are never exposed to the treatment. Moreover, when $\delta_{t}$ from (15) in the SCM model is incorporated into $b_{i}^{\prime} f_{t}$, a general underlying factor model for $Y_{i t}(0)$ as the following can encompass the two methods:

$$
Y_{i t}(0)=\alpha_{i}+b_{i}^{\prime} f_{t}+\beta_{t}^{\prime} \mathbf{X}_{i}+\epsilon_{i t}, \quad i=1, \cdots N, \quad t=1, \cdots T,
$$

where the individual-specific effect $\alpha_{i}$ is not considered in SCM, and the covariate effect $\beta_{t}^{\prime} \mathbf{X}_{i}$ is not included in the original version of $\mathrm{HCW}$, see, for example, Gardeazabal and Vega-Bayo (2016) for details.

However, there are still important differences between SCM and HCW. Wan, Xie and Hsiao (2018) explained these differences carefully and they argued that the main differences between SCM and HCW are the assumptions that each method is based on. HCW places restrictions on 
the control units by assuming that control units must have strong correlation with the treatment unit and should be independent of the treatment, while SCM assumes that the weights must be nonnegative and add up to one, along with (13) and (14) holds approximately. If the HCW assumptions about control units hold for both approaches, the difference between SCM and $\mathrm{HCW}$ is that $\mathrm{HCW}$ is an unconstrained regression, while SCM is a constrained regression. When the constraints are valid, SCM is more efficient. However, when the constraints are invalid, SCM could lead to biased estimation. Therefore, through conducting simulation studies, Wan et al. (2018) found that HCW significantly dominates SCM in a majority of cases.

\section{$\S 5$ Conclusion}

Evaluating the effect of macroeconomic policies quantitatively is one of the central issues in economic studies and policy research in many applied fields. This paper provides a selective review of recent advances in macroeconomic policy evaluation in the framework of the Rubin causal model. Compared to other popular methods such as DSGE and VAR models, the methods proposed by Angrist et al. (2018) and Kuersteiner et al. (2018) do not need to specify the model of the whole economy. Moreover, the dynamic treatment effect exactly corresponds to the impulse function induced from a DSGE or VAR model, which means that the dynamic treatment effect provides an alternative method under less restrictive assumptions. When panel data are available, Hsiao et al. (2012), Abadie and Gardeazabal (2003), and Abadie et al. (2010) provided new methods to estimate the individual treatment effect of macroeconomic policies. In summary, macroeconomic policy evaluation is still a very dynamic and challenging research area deserved further studies. As expected, without doubt, area will receive a great attention in the near future. For example, of importance and challenge is to consider the synthetic control method and/or the HCW model to the quantile treatment effect as in (2).

\section{References}

[1] A Abadie, J D Angrist, GW Imbens. Instrumental variable estimates of the effect of subsidized training on the quantile of trainee earnings. Econometrica, 2002, 70(1): 91-117.

[2] A Abadie, A Diamond, J Hainmueller. Synthetic control methods for comparative case studies: estimating the effect of California s tobacco control program. J Amer Statist Assoc, 2010, 105 (2010): 493-505.

[3] A Abadie, J Gardeazabal. The economic costs of conflict: a case study of the Basque country. Am Econ Rev, 2003, 93(1): 113-132.

[4] A Abadie, GW Imbens. Large sample properties of matching estimators for average treatment effects. Econometrica, 2006, 74(1): 235-267.

[5] A Abadie, GW Imbens. On the failure of the bootstrap for matching estimators. Econometrica, 2008, 76(6): 1537-1557.

[6] A Abadie, G W Imbens. Bias-corrected matching estimators for average treatment effects. J Bus Econ Stat, 2011, 29(1): 1-11. 
Appl. Math. J. Chinese Univ. Vol.*, No*.

[7] A Abadie, GW Imbens. Matching on the estimated propensity score. Econometrica, 2016, 84(2): 781-807.

[8] J H Abbring, G J van den Berg. The nonparametric identification of treatment effects in duration models. Econometrica, 2003, 71(5): 1491-1517.

[9] J D Angrist, G W Imbens. 2-stage least-squares estimation of average causal effects in models with variable treatment intensity. J Am Stat Assoc, 1995, 90(430): 431-442.

[10] J D Angrist, Ò Jordà, G M Kuersteiner. Semiparametric estimates of monetary policy effects: string theory revisited. J Bus Econ Stat, 2018, 36(3): 371-387.

[11] J D Angrist, G M Kuersteiner. Causal effects of monetary shocks: semiparametric conditional independence tests with a multinomial propensity score. Rev Econ Stat, 2011, 93(3): 725-747.

[12] J D Angrist, V Lavy. Using maimonides' rule to estimate the effect of class size on scholastic achievement. Q J Econ, 1999, 114(2): 533-575.

[13] I Bojinov, N Shephard. Time series experiments and causal estimands: exact randomization tests and trading. Social Science Electronic Publishing, 2017.

[14] TLBrunell, JEDiNardo. A propensity score reweighting approach to estimating the Partisan effects of full turnout in American presidential elections. Polit Anal, 2004, 12(1): 28-45.

[15] Z Cai, Y Fang, M Lin, S Tang. Testing conditional unconfoundedness using auxiliary variables. Working paper, Department of Economics, University of Kansas, 2019.

[16] Z Cai, Y Fang, Z Liu. Synergic effects of monetary policy and macro-prudential policy: Based on econometric modeling of macro-policy evaluation. Working paper, Department of Economics, University of Kansas, 2019.

[17] M Caliendo, S Kopeinig. Some practical guidance for the implementation of propensity score matching. J Econ Surv, 2010, 22(1): 31-72.

[18] G Cerulli. Econometric evaluation of socio-economic programs. Springer, 2015.

[19] T Chen, Y Y Ji, Y H,Zhou, P F Zhu. Testing conditional mean independence under symmetry. J Bus Econ Stat, 2014, 36(4):615-627.

[20] V Chernozhukov, C Hansen. An IV model of quantile treatment effects. Econometrica, 2005, 73(1): 245-261.

[21] R H Dehejia, S Wahba. Causal effects in nonexperimental studies: reevaluating the evaluation of training programs. J Am Stat Assoc, 1999, 94(448): 1053-1062.

[22] R H Dehejia, SWahba. Propensity score-matching methods for nonexperimental causal studies. Rev Econ Stat, 2002, 84(1): 151-161.

[23] S G Donald, Y C Hsu, R P Lieli. Testing the unconfoundedness assumption via inverse probability weighted estimators of (l)att. J Bus Econ Stat, 2014, 32(3):395-415.

[24] Z C Du, L Zhang. Home-purchase restriction, property tax and housing price in China: A counterfactual analysis. J Econometrics, 2015, 188(2): 558-568.

[25] H Fujiki, C Hsiao. Disentangling the effects of multiple treatmentsMeasuring the net economic impact of the 1995 great Hanshin-Awaji earthquake. J Econometrics, 2015, 186(1): 66-73. 
Liu Ze-qin, et al. Statistical analysis and evaluation of macroeconomic policies: a selective review

[26] J Gardeazabal, A VegaBayo. An empirical comparison between the synthetic control method and HSIAO et al.'s panel data approach to program evaluation. J Appl Economet, 2017, 32(5): 9831002.

[27] A N Glynn, K M Quinn. An introduction to the augmented inverse propensity weighted estimator. Polit Anal, 2010, 18(1): 36-56.

[28] J Hahn. On the role of the propensity score in efficient semiparametric estimation of average treatment effects. Econometrica, 1998, 66(2): 315-331.

[29] J Hahn, P Todd, W V D Klaauw. Identification and estimation of treatment effects with a regressiondiscontinuity design. Econometrica, 2001, 69(1): 201-209.

[30] J J Heckman, H Ichimura, P E Todd. Matching as an econometric evaluation estimator: evidence from evaluating a job training programme. Rev Econ Stud, 1997, 64(4): 605-654.

[31] J J Heckman, H Ichimura, P E Todd. Matching as an econometric evaluation estimator. Rev Econ Stud, 1998, 65(2): 261-294.

[32] K Hirano, GW Imbens. The propensity score with continuous treatments, in Applied bayesian modeling and causal inference from incomplete-data perspectives, edited by G Andrew and X L Meng, pp.73-84: Wiley-Blackwell, 2004.

[33] K Hirano , GW Imbens, G Ridder. Efficient estimation of average treatment effects using the estimated propensity score. Econometrica, 2003, 71(4): 1161-1189.

[34] P W Holland. Statistics and causal inference. J Am Stat Assoc, 1986, 81(396): 945-960.

[35] D G Horvitz, D J Thompson. A Generalization of Sampling Without Replacement From a Finite Universe. J Am Stat Assoc, 1952, 47(260): 663-685.

[36] C Hsiao, HS Ching, S K Wan. A panel data approach for program evaluation: measuring the benefits of political and economic integration of Hong Kong with Mainland China. J Appl Economet, 2012, 27(5): 705-740.

[37] G W Imbens. The role of the propensity score in estimating dose-response functions. Biometrika, 2000, 87(3): 706-710.

[38] G W Imbens. Nonparametric estimation of average treatment effects under exogeneity: a review. Rev Econ Stat, 2004, 86(1): 4-29.

[39] G W Imbens, J M Wooldridge. Recent developments in the econometrics of program evaluation. J Econ Lit, 2009, 47(1): 5-86.

[40] Ò Jordà, A M Taylor. The time for austerity: estimating the average treatment effect of fiscal policy. Econ J, 2016, 126(590): 219-255.

[41] J D Y Kang, J L Schafer. Demystifying Double Robustness: A Comparison of Alternative Strategies for Estimating a Population Mean from Incomplete Data. Stat Sci, 2007, 22(4): 523-539.

[42] W V D Klaauw. Breaking the link between poverty and low student achievement: An evaluation of Title I. J Econometrics, 2008, 142(2): 731-756.

[43] R Koenker, G Bassett. Regression quantiles. Econometrica, 1978, 46(1): 33-50.

[44] G M Kuersteiner, D C Phillips, M Villamizar-Villegas. Effective sterilized foreign exchange intervention? Evidence from a rule-based policy. J Int Econ, 2018, 113: 118-138. 
Appl. Math. J. Chinese Univ. Vol.*, No*.

[45] M J van der Lann, J M Robins. Unified methods for censored longitudinal data and causality. Springer, 2003.

[46] D S Lee. Randomized experiments from non-random selection in U.S. house elections. J Econometrics, 2008, 142(2): 675-697.

[47] D S Lee, D Card. Regression discontinuity inference with specification error. J Econometrics, 2006, 142(2): 655-674.

[48] K T Li, D R Bell. Estimation of average treatment effects with panel data: Asymptotic theory and implementation. J Econometrics, 2017, 197(1): 65-75.

[49] J J Lok. Statistical modeling of causal effects in continuous time. Ann Stat, 2008, 36(3): 14641507.

[50] J Ludwig, D L Miller. Does head start improve children's life chances? evidence from a regression discontinuity design. Q J Econ, 2007, 122(1): 159-208.

[51] J K Lunceford, M Davidian. Stratification and weighting via the propensity score in estimation of causal treatment effects: a comparative study, Stat Med, 2004, 23(19): 2937-2960.

[52] A Niculescu-Mizil, R Caruana. Predicting good probabilities with supervised learning. Proceedings of the 22nd international conference on Machine learning, Bonn, Germany, 2005: 625-632.

[53] M Ouyang, Y Peng. The treatment-effect estimation: a case study of the 2008 economic stimulus package of China. J Econometrics, 2015, 188(2): 545-557.

[54] F Pedregosa et al. Scikit-learn: Machine Learning in Python. J Mach Learn Res, 2011, 12: $2825-2830$.

[55] J M Robins. A new approach to causal inference in mortality studies with a sustained exposure periodapplication to control of the healthy worker survivor effect, Mathl Modelling, 1986, 7(9): 1393-1512.

[56] J M Robins. Correcting for non-compliance in randomized trials using structural nested mean models Commun Stat-Theor M, 1994, 23(8): 2379-2412.

[57] J M Robins, S Greenland, F C Hu. Estimation of the causal effect of a time-varying exposure on the marginal mean of a repeated binary outcome. J Am Stat Assoc, 1999, 94(447): 687-700.

[58] J M Robins, A Rotnitzky. Semiparametric efficiency in multivariate regression models with missing data. J Am Stat Assoc, 1995, 90(429): 122-129.

[59] J M Robins, A Rotnitzky, L P Zhao. Analysis of semiparametric regression models for repeated outcomes in the presence of missing data. J Am Stat Assoc, 1995, 90(429): 106-121.

[60] P R Rosenbaum, D B Rubin. The central role of the propensity score in observational studies for causal effects. Biometrika, 1983, 70(1): 41-55.

[61] D B Rubin. Matching to remove bias in observational studies. Biometrics, 1973a, 29(1): 159-183.

[62] D B Rubin. The use of matched sampling and regression adjustment to remove bias in observational studies. Biometrics, 1973b, 29(1): 185-203.

[63] D B Rubin. Estimating causal effects of treatments in randomized and nonrandomized studies. J Educ Psychol, 1974, 66(5): 688-701. 
[64] D B Rubin. Assignment to Treatment Group on the Basis of a Covariate. J Educ Stat, 1977, 2(1): $1-26$.

[65] D B Rubin. Bayesian inference for causal effects: the role of randomization. Ann Stat, 1978, 6(1): $34-58$.

[66] D B Rubin. Using multivariate matched sampling and regression adjustment to control bias in observational studies. J Am Stat Assoc, 1979, 74(366): 318-328.

[67] DL Thistlethwaite, D T Campbell. Regression-discontinuity analysis: an alternative to the ex post facto experiment. J Educ Psychol, 1960, 51(6): 309-317.

[68] S K Wan, Y Xie, C Hsiao. Panel data approach vs synthetic control method. Econ Lett, 2018, 164: 121-123.

[69] J M Wooldridge. Inverse probability weighted estimation for general missing data problems, J Econometrics, 2007, 141(2): 1281-1301.

[70] J M Wooldridge. Econometric analysis of cross section and panel data, MIT Press, 2010.

${ }^{1}$ The Wang Yanan Institute for Studies in Economics, MOE Key Laboratory of Econometrics (Xiamen University), Fujian Provincial Key Laboratory of Statistical Science, Xiamen University, Xiamen 361005, China. E-mail: yifst1@xmu.edu.cn.

${ }^{2}$ Department of Economics, University of Kansas, Lawrence, KS 66045, USA.

${ }^{3}$ Department of Statistics, School of Economics, Xiamen University, Xiamen 361005, China. 